Article

\title{
Eucalyptus gunnii and Eucalyptus pulverulenta 'Baby Blue' Essential Oils as Potential Natural Herbicides
}

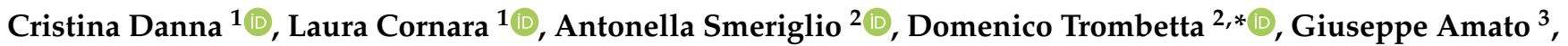 \\ Pierluca Aicardi ${ }^{4}$, Laura De Martino ${ }^{3, *}$, , Vincenzo De Feo $^{3(\mathbb{D})}$ and Lucia Caputo ${ }^{3}$ (D)
}

1 Department of Earth, Environment and Life Sciences, University of Genova, 16132 Genova, Italy; cristina.danna@edu.unige.it (C.D.); laura.cornara@unige.it (L.C.)

2 Department of Chemical, Biological, Pharmaceutical and Environmental Sciences, University of Messina, 98166 Messina, Italy; asmeriglio@unime.it

3 Department of Pharmacy, University of Salerno, 84084 Fisciano, Italy; giuseppe.amato119@gmail.com (G.A.); defeo@unisa.it (V.D.F.); lcaputo@unisa.it (L.C.)

4 Pierluca Aicardi, Coldiretti Savona, 17100 Savona, Italy; pierluca.aicardi@coldiretti.it

* Correspondence: dtrombetta@unime.it (D.T.); ldemartino@unisa.it (L.D.M.); Tel.: +39-09-0676-6458 (D.T.); +39-08-996-8292 (L.D.M.)

\section{check for}

updates

Citation: Danna, C.; Cornara, L.; Smeriglio, A.; Trombetta, D.; Amato, G.; Aicardi, P.; De Martino, L.; De Feo, V.; Caputo, L. Eucalyptus gunnii and Eucalyptus pulverulenta 'Baby Blue' Essential Oils as Potential Natural Herbicides. Molecules 2021, 26, 6749 https://doi.org/10.3390/ molecules26216749

Academic Editor:

Francesca Mancianti

Received: 4 October 2021

Accepted: 4 November 2021

Published: 8 November 2021

Publisher's Note: MDPI stays neutral with regard to jurisdictional claims in published maps and institutional affiliations.

Copyright: (c) 2021 by the authors. Licensee MDPI, Basel, Switzerland. This article is an open access article distributed under the terms and conditions of the Creative Commons Attribution (CC BY) license (https:// creativecommons.org/licenses/by/ $4.0 /)$.
Abstract: The phytotoxicity and eco-compatibility of essential oils (EOs) from Eucalyptus gunnii (EG) and E. pulverulenta 'Baby Blue' (EP), cultivated in Italy for their cut foliage, were investigated. Leaf micromorphology, EOs phytochemical characterization, and phytotoxicity were analysed. EP revealed a significantly higher oil gland density and a higher EO yield with respect to EG. In both EOs, 1,8-cineole was the major compound ( 75\%), followed by $\alpha$-pinene in EG (13.1\%) and eugenol in EP (7.5\%). EO phytotoxicity was tested on both weeds (Lolium multiflorum, Portulaca oleracea) and crops (Raphanus sativus, Lactuca sativa, Lepidium sativum, Solanum lycopersicum, Pisum sativum, Cucumis sativus). EG EO inhibited germination of P. oleracea, R. sativus, and S. lycopersicum seeds (ranging from 61.5 to $94.6 \%$ for the higher dose used), while affecting only radical elongation in S. lycopersicum (ranging from 66.7 to $82.6 \%$ ). EP EO inhibited germination of P. oleracea and R. sativus (ranging from 41.3 to $74.7 \%$ ) and affected radical elongation of L. sativum and L. multiflorum (ranging from 57.4 to $76.0 \%$ ). None of the EOs affected the germination and radical growing of L. sativa, P. sativum, and C. sativus. Moreover, EP EO was more active than EG EO in inhibiting $\alpha$-amylase, a key enzyme for seed growth regulation. Brine shrimp lethality assay showed that both EOs are safe for aquatic organisms, suggesting their high eco-compatibility. The data collected provide useful information for future applications of these EOs in agriculture as safe and selective bioherbicides.

Keywords: Eucalyptus; oil cavities; micromorphology; essential oils; natural herbicides; phytotoxicity; $\alpha$-amylase; eco-compatibility

\section{Introduction}

Native from Australia, the genus Eucalyptus (Myrtaceae) contains about 800 species and is largely cultivated around the world in commercial plantations for several applications, such as cellulose, pulp, gum, essential oils, and honey production, as well as for construction and as ornamental plants [1,2].

In Italy, several Eucalyptus species were introduced at the beginning of the 1800 s by plant collectors to be used as ornamental trees in private gardens [3]. During the early 1900s, these trees became largely cultivated, especially in South Italy, for reclaiming swampy lands and eliminating the outbreak of malaria [4]. Today, the Eucalyptus genus is still cultivated in many Italian regions, growing until $350 \mathrm{~m}$ above sea level. Long-established eucalyptus cut foliage plantations occur in Imperia and Western Liguria (Italy), in the Alpes Maritime (France), and in Cornwall (England), while less extensive plantations are also present in Ireland [5]. Nowadays, Italy is the leader in Europe in terms of the production and export 
of eucalyptus cut foliage, which is mainly sold on the Northern European market, where fresh and dried branches are used in floral compositions [6].

In Liguria, different species are cultivated for this purpose, such as E. cinerea F. Muell. ex. Benth., E. gunnii Hook.f., E. pulverulenta Sims cv 'Baby blue', and E. parvula L.A.S. Johnson and K.D.Hill. Correct pruning is useful to produce high quality juvenile stems from Eucalyptus species [7]. During harvesting, stems are carefully selected for the quality of branches based on the color and shape of the foliage. As a result, a large amount of waste biomass is produced. It is estimated that, in the Province of Savona, Western Liguria, where these plantations have an extension of $100 \mathrm{ha}$, a total of about 1000 tons per year of cut foliage is produced, with waste of more than $4 \%$ (4 tons), which is currently buried or burnt (Coldiretti, Savona; personal communication).

However, this discarded plant material is still rich in bioactive compounds with interesting biological properties. Recent studies have shown the potential of remnants from Eucalyptus pruning for transformation into added value products $[8,9]$. The genus Eucalyptus is characterized by the presence of many secondary compounds, such as phenolics, essential oils, waxes, and resins. The abundance of bioactive compounds suggests different applications, such as pharmaceutics, cosmetics, natural pesticides, insecticides, and herbicides [10].

The use of synthetic compounds for pest control has harmed the environment and human health. Glyphosate-based formulations, extensively used as herbicide worldwide, have been recently referred to as 'probably carcinogenic' [11]. For this reason, together with the development of resistance in weeds and pathogens, many countries have recently restricted or banned the use of such formulations [12]. Therefore, essential oils (EOs) showing bioherbicidal potential and not being persistent in the environment represent a valuable alternative to synthetic chemicals in integrated weed management. This possibility is of particular interest also considering that, in Eucalyptus plantations, the ground is still maintained weed-free using glyphosates, such as Roundap (Bayer). Eucalyptus EOs, rich in 1,8-cineole, can play an important role in modern agriculture to reduce the use of harmful herbicides and pesticides thanks to their allelopathic effect on weeds and to the interaction with noxious insects [13-15].

Phytotoxic effects of different Eucalyptus EOs have been reported on many common weed species. E. globulus EO showed significant effects on seed germination and seedling growth of Amaranthus blitoides, Cynodon dactylon [16,17], and Chenopodium album [18]. One of the main components of Eucalyptus EOs, eugenol, significantly inhibited the germination of Cassia occidentalis and Bidens pilosa at a very low concentration [19]. E. citriodora EO significantly affected both the radical and hypocotyl development of Lolium multiflorum [20], while E. camaldulensis EO completely inhibited seed germination of Amaranthus hybridus and Portulaca oleracea [21]. However, these EOs can also cause damage to some crops, making it essential to assess their selectivity $[22,23]$. The phytotoxicity on weeds is probably due to the alteration of several biochemical and physiological processes, affecting seed germination and/or hypocotyl and radical elongation $[19,20,24,25]$. These activities depend on the quantitative and qualitative composition of the $\mathrm{EO}$ of each individual Eucalyptus species. Therefore, the identification of the species source of EOs is important, especially when pruning material comes from mixed plantations, where different species are found side by side. Micro-morphological analyses are an essential starting point of quality control, especially for plant remnants and by-products [26]. These analyses involve the use of light microscopy (LM) and scanning electron microscopy (SEM), allowing to characterize the plant material, highlight contaminations [27], and establish the botanical identity.

We carried out the micro-morphological characterization of pruning remnants from two different Eucalyptus species, namely E. gunnii and E. pulverulenta 'Baby blue', cultivated for cut foliage in the hinterland of Western Liguria (Italy). In addition, the phytochemical profile of the EOs obtained from the biomass of these plants was analyzed and tested for selective phytotoxicity on weeds and crop seeds. Finally, their eco-compatibility was 
evaluated for safe applications in agriculture as bioherbicides, according to the principles of a circular economy.

\section{Results and Discussion}

\subsection{Micromorphological Studies}

Micromorphological analyses were performed on juvenile leaves of E. gunnii (EG) and E. pulverulenta 'Baby blue' (EP) collected during January 2021 in the hinterland of Western Liguria (Italy) (Figure 1A-D). EG shows dark green leaves, varying from sessile and rounded to peduncolate and more elongated (Figure 1B), while the EP blue-gray leaves are sessile with a rounded shape (Figure 1C).
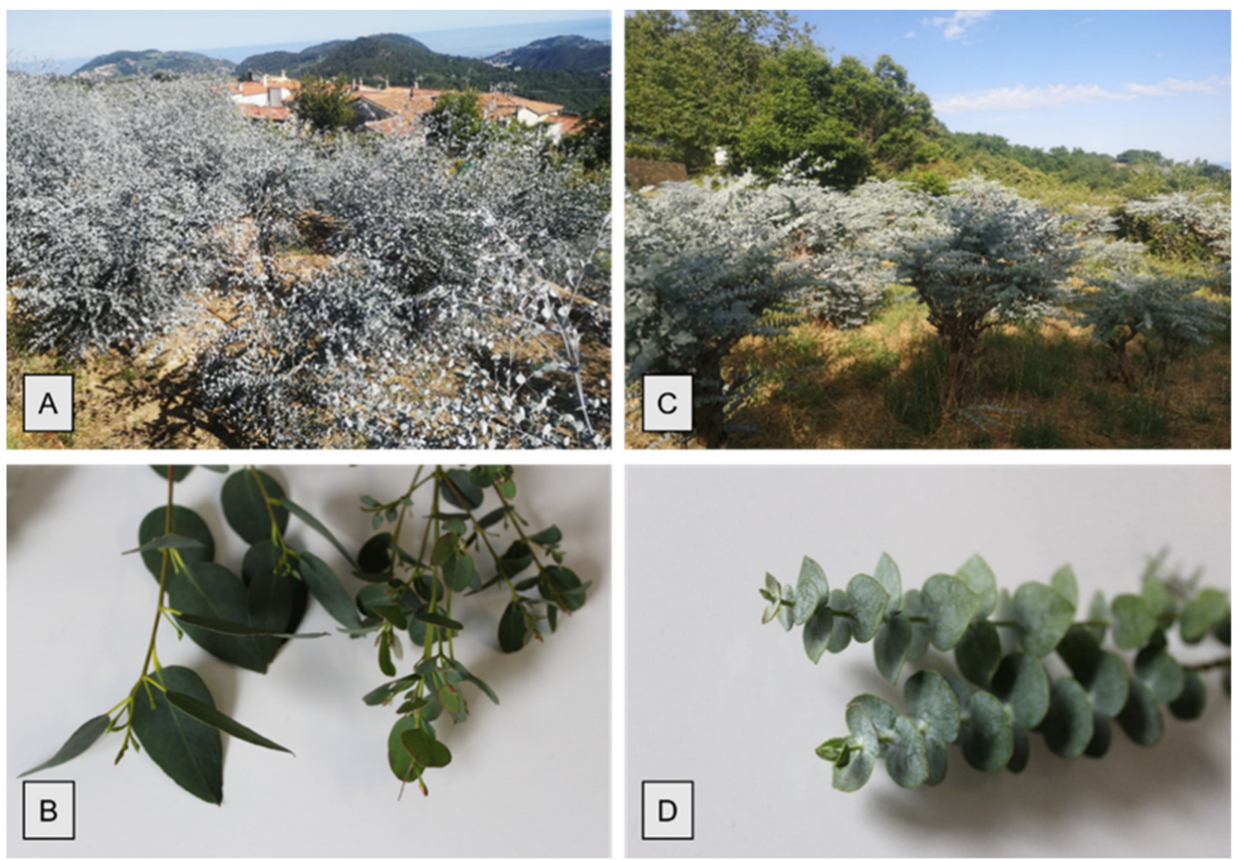

Figure 1. Plantation and branches of E. gunnii (A,B) and of E. pulverulenta 'Baby blue' (C,D).

The leaf surface of both species is glabrous, leathery, and waxy and shows many oil glands, giving them a dotted appearance (Figure 2). The venation pinnate-reticulate is slightly evident in EG (Figure 2A), while it appears much more prominent in EP (Figure 2B). Similarly, a larger wax deposition results in a more intense powdery blue-grey surface in EP (Figure 2B).
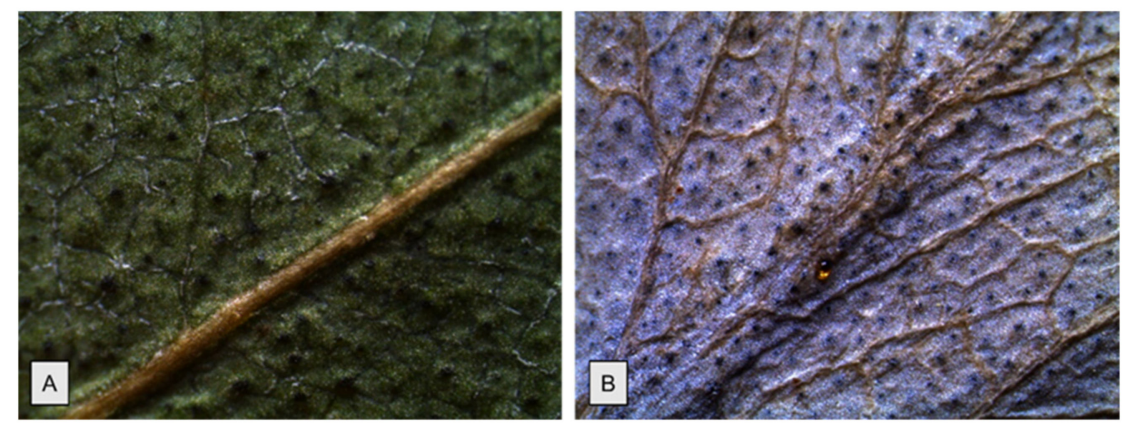

Figure 2. Abaxial surfaces of E. gunnii (A) and of E. pulverulenta 'Baby blue' (B) at $2.5 \times$ magnification.

The observation by both light microscopy (LM) and scanning electron microscopy (SEM) showed in both species the amphistomatal leaves with anomocytic stomata, oval or rounded, appearing at the same level as the neighbouring epidermal cells and as the overlying cells surrounding oil cavities (Figure 3A-H and Figure 4A-D). These features 
agree with data previously reported for other Eucalyptus species $[28,29]$. Trichomes are absent, as also reported for E. cinerea F. Muell. ex Benth. by Soliman and coworkers [30].
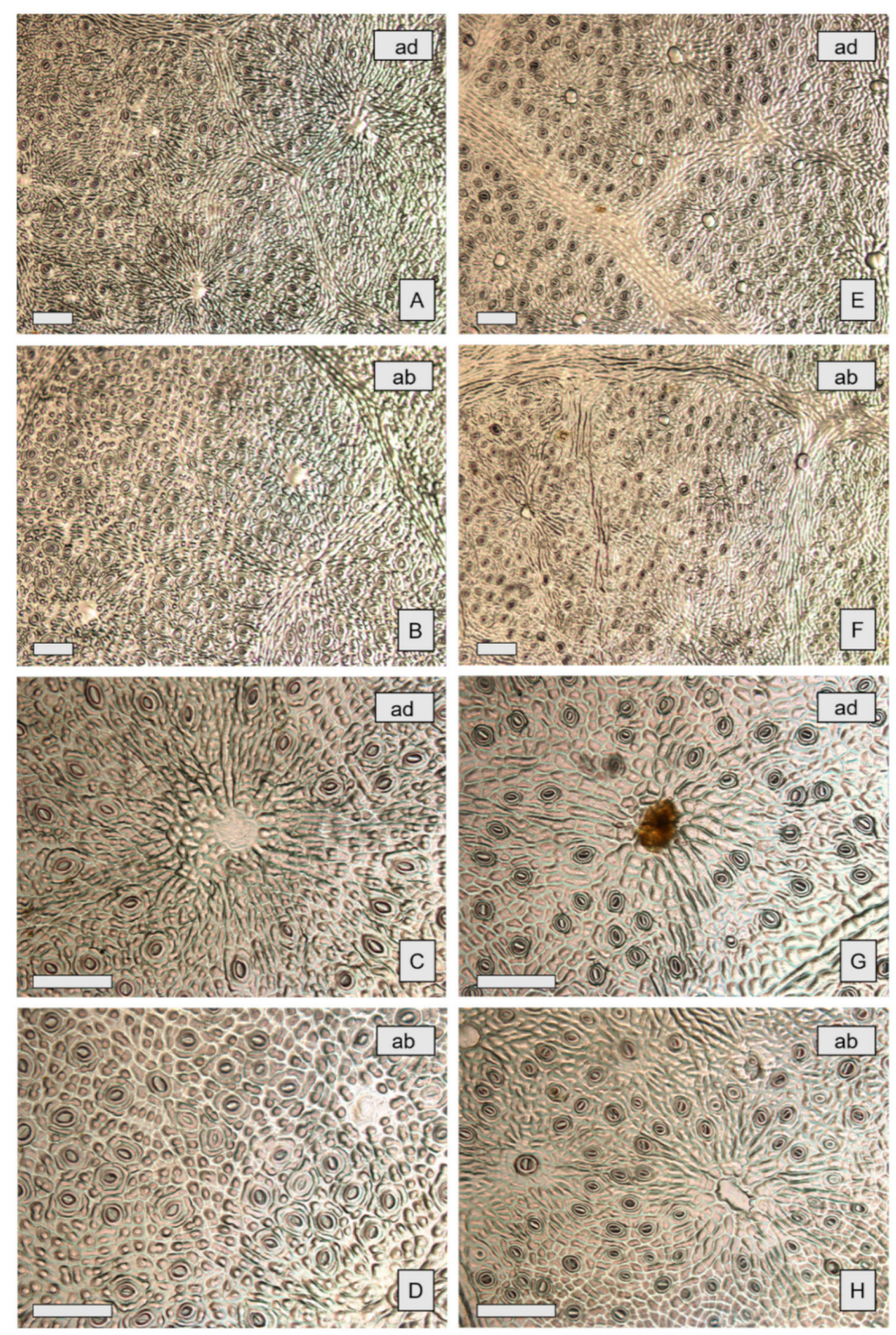

Figure 3. LM-A-D: E. gunnii (EG); E-H: E. pulverulenta 'Baby blue' (EP). Comparison between leaf peelings from abaxial (ab) and adaxial surfaces (ad), at different magnifications. EG: adaxial surface at $10 \times(\mathbf{A})$ and at $20 \times(\mathbf{C})$; abaxial surface at $10 \times(\mathbf{B})$ and at $20 \times(\mathbf{D})$. EP: adaxial surface at $10 \times$ (E) and at $20 \times(\mathbf{G})$; abaxial surface at $10 \times(\mathbf{F})$ and at $20 \times(\mathbf{H})$. Scale bars: $\mathrm{A}-\mathrm{H}=100 \mu \mathrm{m}$. 

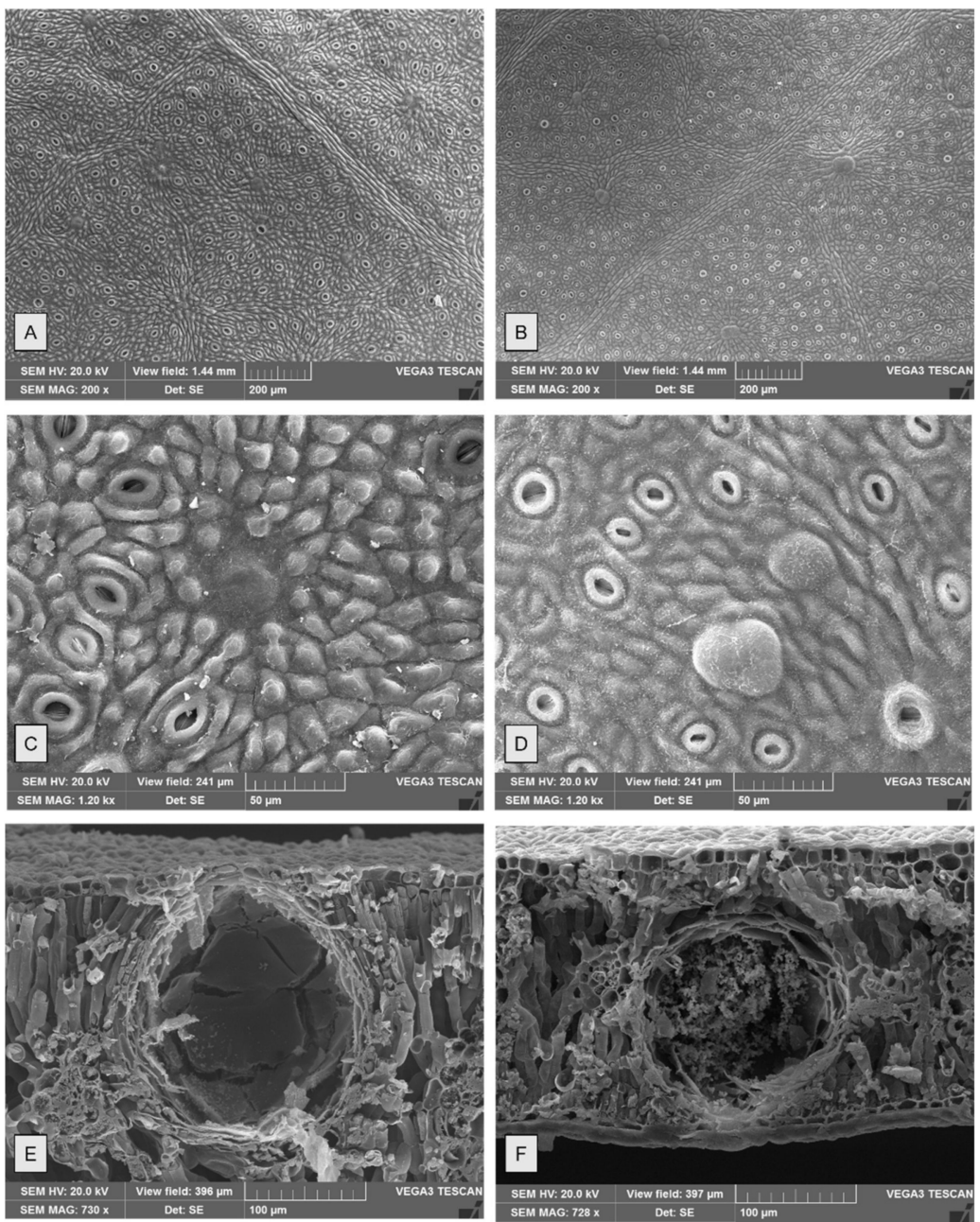

Figure 4. SEM-Comparison between E. gunnii (A,C,E) and E. pulverulenta 'Baby blue' (B,D,F) leaves. Adaxial surfaces at $200 \times(\mathbf{A}, \mathbf{B})$. Overlying cells surrounding oil cavities in the abaxial surfaces, at $1200 \times(\mathbf{C}, \mathbf{D})$. Leaf transversal sections showing oil cavities, containing amorphous material $(\mathbf{E}, \mathbf{F})$.

Epidermal peelings showed amorphous yellow oil content, sometimes spread out among the cavities (Figure 3G). Epidermal cells in both species are polygonal isodiametric or slightly elongated in shape. Numerous papillae were observed in EG (Figure 4C), while they were not visible in EP (Figure 4D). Significant differences in glands' density were found among the two species, with EP showing a higher mean gland density (Table 1). In addition, in each species, significant differences in gland density were also found between adaxial and abaxial surfaces of the leaf (Figure 3A,B,E-F and Figure 4A,B), as shown in Table 1.

Table 1. E. gunnii (EG) and E. pulverulenta 'Baby blue' (EP) oil gland mean density (glands $\mathrm{cm}^{-2}$ ) and mean dimensions $(\mu \mathrm{m})$.

\begin{tabular}{ccccc}
\hline \multirow{2}{*}{ Species } & \multicolumn{2}{c}{$\begin{array}{c}\text { Oil Gland Mean Density } \\
(\text { Glands cm }\end{array}$} & \multicolumn{2}{c}{$\begin{array}{c}\text { Oil Gland Mean Dimensions } \\
( \pm \text { S.D.) }\end{array}$} \\
\hline & Abaxial & Adaxial & Height & Width \\
E. gunnii & $213( \pm 66)$ & $416( \pm 116)$ & $199( \pm 34)$ & $161( \pm 31)$ \\
E. pulverulenta & $750( \pm 96)$ & $1321( \pm 176)$ & $188( \pm 32)$ & $142( \pm 26)$ \\
\hline
\end{tabular}

The data of gland density and dimensions are expressed as mean \pm S.D. Oil gland density. EG: abaxial vs. adaxial, $p<0.01, n=12$; EP: abaxial vs. adaxial, $p<0.01, n=12$; EG: abaxial vs. EP abaxial, $p<0.01, n=12$ : EG: adaxial vs. EP adaxial, $p<0.01, n=12$. Oil gland dimensions. EG vs. EP height and width, $p>0.05, n=21$. $t$-test was used for comparisons of the micromorphological data. The results were considered significant for $p<0.01$. 
Similar data on oil gland density have been reported for another species, namely $E$. polybractea F. Muell. ex R.T. Baker [31]. However, the number and the size of oil glands vary among the species and, in some cases, it is very high, such as in E. protensa, showing up to 3400 oil glands $\mathrm{cm}^{-2}$ [32].

In leaf transversal sections (Figure 5), the oil glands appear distributed throughout the mesophyll and are more protruding in the upper surface in both species. The oil cavities cut close to the gland midpoint appeared spherical/ellipsoidal or pear-shaped. No significant differences were found between gland dimensions of the two species (Table 1). Each gland had a single internal epithelial layer, and the amorphous content was visible within the cavity (Figure 4E,F and Figure 5C,D).

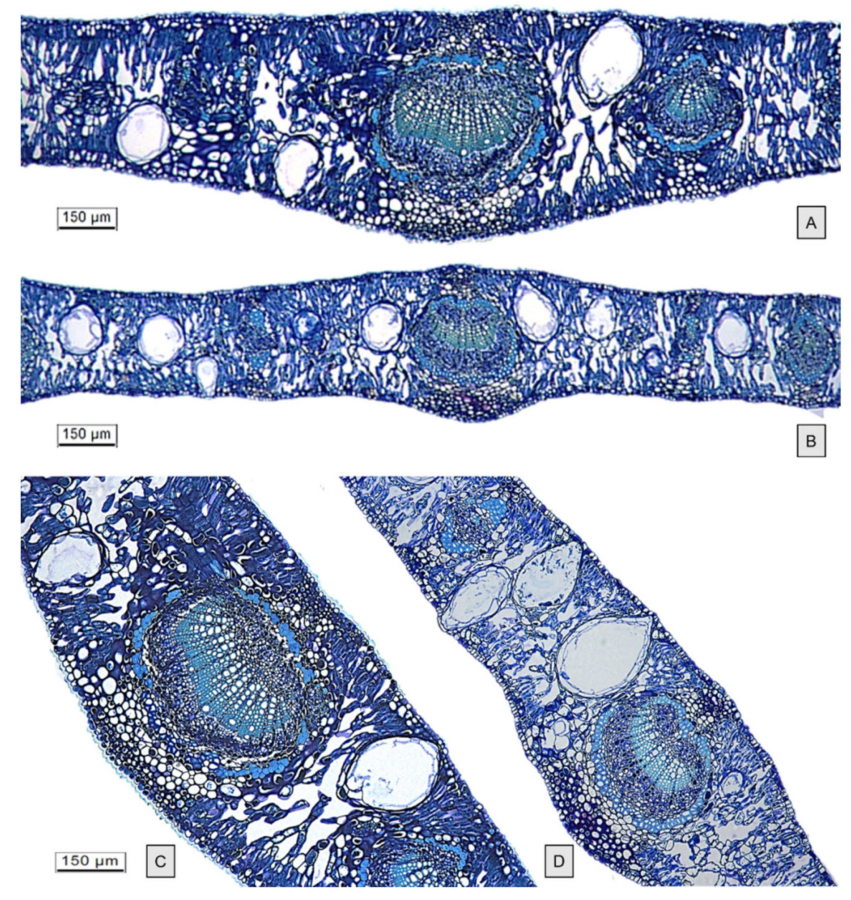

Figure 5. LM-TBO staining. Transverse sections through leaf midrib of E. gunnii (EG) (A-C) and E. pulverulenta 'Baby blue' (EP) (B-D), showing oil cavities varying from spherical/ellipsoidal to pear-shaped.

\subsection{Chemical Composition of Essential Oils}

The hydrodistillation of the aerial parts of EG and EP furnished pale yellow EOs at a yield of 1.1 and $1.6 \%$ on a dry mass basis, respectively. The yield of the two EOs is an important parameter to consider, because they derive from waste material and can be useful in the perspective of a circular economy. These data agree with the micromorphological observations on the leaves from the two species, showing a lower gland density in EG with respect to that in EP. Figure 6A,B shows the chromatograms of the two EOs. The EOs composition with retention indices and area percentages for each compound are reported in Table 2; the compounds are listed according to their elution order on a HP-5MS column. Altogether, 37 compounds were identified, 30 for EG, accounting for $98.1 \%$ of the total EO, and 25 for EP, accounting for $97.7 \%$ of the total $\mathrm{EO}$. The oxygenated monoterpenes are the main constituents in both EOs, with a percentage of $80.3 \%$ for EG and $79.1 \%$ for EP. 


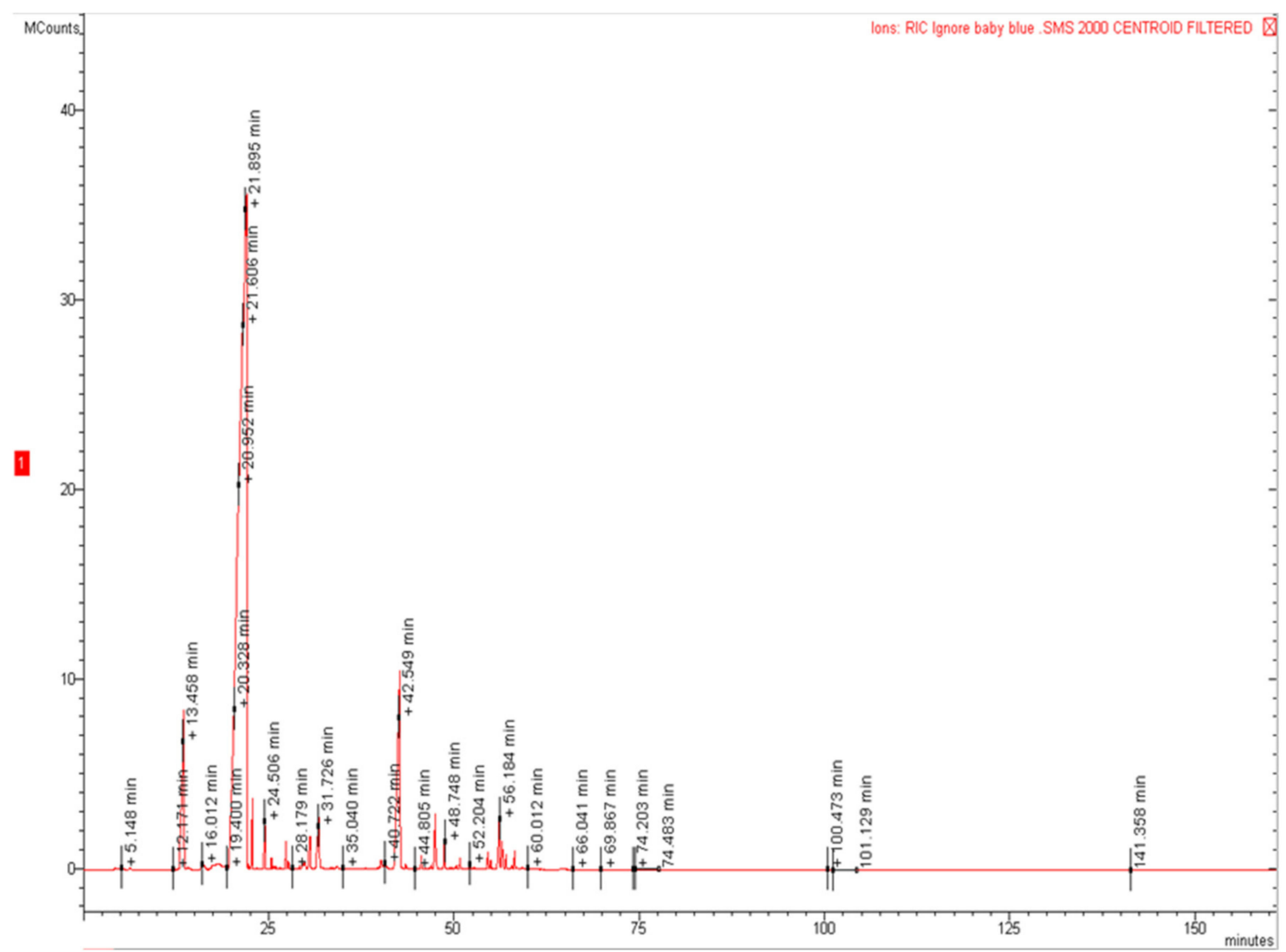

(A)

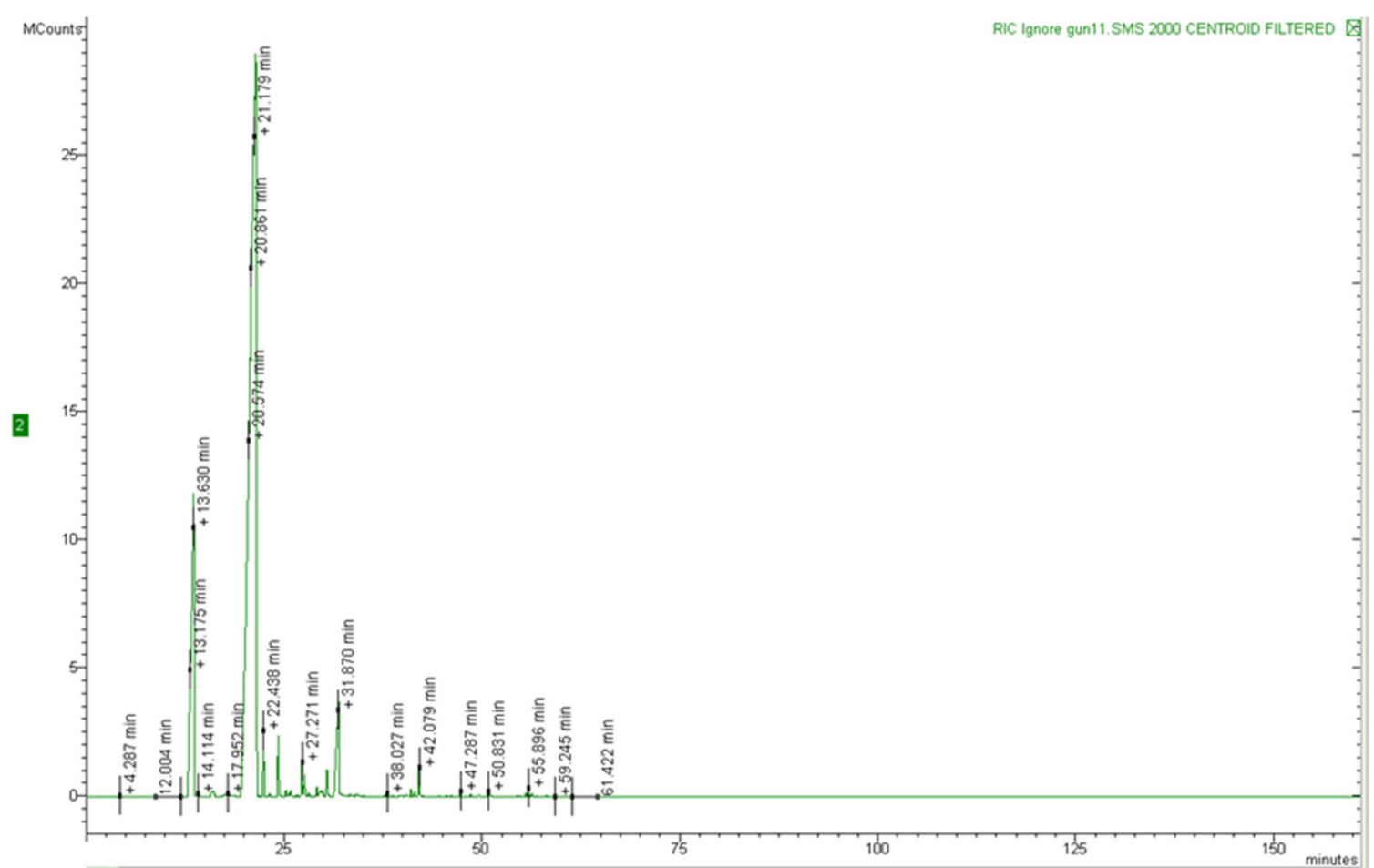

(B)

Figure 6. GC chromatograms of E. pulverulenta 'Baby blue' (EP) (A) and E. gunnii (EG) (B) EOs. 
Table 2. Chemical composition of E. gunnii (EG) and E. pulverulenta 'Baby blue' (EP) EOs.

\begin{tabular}{|c|c|c|c|c|c|c|c|}
\hline & RT & Compound & \multicolumn{2}{|c|}{$\%$} & \multirow[t]{2}{*}{$\mathrm{KI}^{\mathrm{a}}$} & \multirow[t]{2}{*}{$\mathrm{KI}^{\mathrm{b}}$} & \multirow[t]{2}{*}{ Identification $^{c}$} \\
\hline & & & EG & EP & & & \\
\hline 1 & 5.148 & $\begin{array}{l}\text { 1-Methyl-1,3- } \\
\text { cyclohexadiene }\end{array}$ & & $\mathrm{t}$ & 759 & 1183 & 1,2 \\
\hline 2 & 5.267 & $\begin{array}{l}\text { 3-Methyl-2- } \\
\text { buten-1-ol }\end{array}$ & & $\mathrm{t}$ & 761 & 1328 & 1,2 \\
\hline 3 & 6.246 & n-Octane & & $\mathrm{t}$ & 773 & & 1,2 \\
\hline 4 & 7.299 & (2Z)-Hexenol & & $\mathrm{t}$ & 787 & & 1,2 \\
\hline 5 & 12.004 & $\begin{array}{l}\text { Santolina } \\
\text { triene }\end{array}$ & $\mathrm{t}$ & & 848 & 1043 & 1,2 \\
\hline 6 & 13.849 & $\alpha$-Pinene & 13.1 & 4.8 & 872 & 1028 & $1,2,3$ \\
\hline 7 & 14.036 & Camphene & 0.1 & 0.1 & 874 & 1075 & $1,2,3$ \\
\hline 8 & 16.071 & $\beta$-Pinene & 0.3 & 0.5 & 901 & 1120 & $1,2,3$ \\
\hline 9 & 16.945 & $\begin{array}{c}\text { dehydro-1,8 } \\
\text { Cineole }\end{array}$ & $\mathrm{t}$ & & 912 & 1095 & 1,2 \\
\hline 10 & 17.952 & Myrcene & 0.3 & 0.4 & 925 & 1173 & $1,2,3$ \\
\hline 11 & 18.695 & $\delta$-2-Carene & 0.1 & 0.2 & 934 & 1146 & $1,2,3$ \\
\hline 12 & 19.400 & $\begin{array}{c}\beta- \\
\text { Phellandrene }\end{array}$ & & $\mathrm{t}$ & 944 & 1206 & $1,2,3$ \\
\hline 13 & 19.500 & p-Cymene & $\mathrm{t}$ & & 945 & 1279 & $1,2,3$ \\
\hline 14 & 20.574 & 1,8-Cineole & 74.7 & 75.5 & 959 & 1220 & $1,2,3$ \\
\hline 15 & 21.870 & (Z)- $\beta$-Ocimene & $t$ & & 976 & 1240 & $1,2,3$ \\
\hline 16 & 22.438 & (E)- $\beta$-Ocimene & 1.9 & 1.0 & 983 & 1260 & $1,2,3$ \\
\hline 17 & 25.243 & $\gamma$-Terpinene & 0.3 & 0.9 & 1016 & 1254 & $1,2,3$ \\
\hline 18 & 27.271 & $\begin{array}{l}\text { dehydro- } \\
\text { Linalool }\end{array}$ & 0.5 & 0.5 & 1043 & & 1,2 \\
\hline 19 & 27.644 & $\begin{array}{c}1,3,8-p- \\
\text { Menthatriene }\end{array}$ & 0.4 & & 1048 & & 1,2 \\
\hline 20 & 28.128 & allo-Ocimene & 0.1 & & 1055 & 1382 & 1,2 \\
\hline 21 & 28.799 & neo-Isopulegol & $t$ & & 1064 & & 1,2 \\
\hline 22 & 29.513 & Borneol & 0.2 & & 1074 & 1715 & $1,2,3$ \\
\hline 23 & 29.776 & endo-Fenchol & 0.2 & 1.0 & 1077 & & 1,2 \\
\hline 24 & 31.870 & Terpineol & 4.2 & 1.5 & 1099 & 1710 & $1,2,3$ \\
\hline 25 & 32.268 & cis-Verbenol & 0.2 & 0.1 & 1104 & 1663 & 1,2 \\
\hline 26 & 34.118 & Verbenone & 0.2 & 0.2 & 1131 & & 1,2 \\
\hline 27 & 39.679 & Thymol & 0.1 & 0.3 & 1205 & 2172 & $1,2,3$ \\
\hline 28 & 40.403 & $\delta$-Elemene & 0.1 & & 1216 & 1460 & $1,2,3$ \\
\hline 29 & 41.032 & $\gamma$-Elemene & 0.1 & & 1226 & 1651 & $1,2,3$ \\
\hline 30 & 41.512 & $\begin{array}{l}\text { Myltayl-4(12)- } \\
\text { ene }\end{array}$ & 0.1 & & 1233 & & 1,2 \\
\hline 31 & 42.079 & $\begin{array}{l}\text { dihydro- } \\
\text { Eugenol }\end{array}$ & 0.5 & 7.5 & 1242 & & 1,2 \\
\hline 32 & 43.444 & $\alpha$-Copaene & $\mathrm{t}$ & 0.1 & 1262 & 1477 & $1,2,3$ \\
\hline 33 & 45.520 & Sibirene & $\mathrm{t}$ & 0.7 & 1294 & & 1,2 \\
\hline 34 & 46.066 & Caryophyllene & 0.2 & & 1296 & 1607 & $1,2,3$ \\
\hline 35 & 50.831 & $\begin{array}{c}\alpha \text {-Guaiene } \\
\text { Cycloisolongifol- }\end{array}$ & 0.2 & 0.3 & 1372 & 1583 & 1,2 \\
\hline 36 & 52.204 & $\begin{array}{c}5-\text { ol } \\
<\text { trans> }\end{array}$ & & 0.2 & 1395 & & 1,2 \\
\hline \multirow[t]{6}{*}{37} & 56.184 & Globulol & & 1.9 & 1457 & 2095 & 1,2 \\
\hline & & Total & 98.1 & 97.7 & & & \\
\hline & & $\begin{array}{l}\text { Monoterpene } \\
\text { hydrocarbons }\end{array}$ & 17.1 & 15.4 & & & \\
\hline & & $\begin{array}{l}\text { Oxygenated } \\
\text { monoterpenes }\end{array}$ & 80.3 & 79.1 & & & \\
\hline & & $\begin{array}{l}\text { Sesquiterpenes } \\
\text { hydrocarbons }\end{array}$ & 0.7 & 1.1 & & & \\
\hline & & $\begin{array}{l}\text { Oxygenated } \\
\text { sesquiterpenes }\end{array}$ & & 2.1 & & & \\
\hline
\end{tabular}

$\mathrm{a}, \mathrm{b}$ The Kovats retention indices determined relative to a series of n-alkanes $\left(\mathrm{C}_{10}-\mathrm{C}_{35}\right)$ on the apolar HP-5 MS and the polar HP Innowax capillary columns, respectively; ${ }^{\mathrm{C}}$ identification method: $1=$ comparison of the Kovats retention indices with published data, $2=$ comparison of mass spectra with those listed in the NIST 02 and Wiley 275 libraries and with published data, and $3=$ coinjection with authentic compounds; $\mathrm{t}=$ trace $(<0.1 \%)$.

1,8-Cineole was the main constituent in both EOs, with a percentage of 74.7\% in EG and $75.5 \%$ in EP. Other components present in a lesser amount in EG were $\alpha$-pinene $(13.1 \%)$ and terpineol (4.2\%), while in EP, they were dihydro-eugenol (7.5\%) and $\alpha$-pinene (4.8\%). 
1,8-Cineole was reported as the main constituent of EG EO also in the samples from Argentina (17.9\%); Serbia (67.8\%); and Sardinia, Italy (33.0\%), even if with a different percentage on total EO [33-35]. Regarding the other constituents, the Argentinian EO presented high percentages of $p$-cymene (12.3\%), spathulenol (12.3\%), and $\alpha$-phellandrene (7.0\%) [33], as well as from the Sardinian EO that showed high amounts of trans-sabinene hydrate acetate (15\%), globulol (10.3\%), and longicyclene (9.1\%) [35]. Only the EO from Serbia presented $\alpha$-pinene as the second major compound with a percentage of $14.1 \%$, similar to that of the sample here presented [34]. Elaissi and coworkers [36] reported a Tunisian EG EO with a higher percentage of oxygenated sesquiterpenes (spathulenol, globulol, and viridiflorol) with respect to EO here reported, rich in oxygenated monoterpenes.

Few studies reported the chemical composition of EP EO [37-39]. The chemical composition of our sample agrees with those reported in these previous studies: 1,8cineole was the main constituent, ranging from 75.1 to $85.1 \%$, and $\alpha$-pinene was present in comparable amounts with respect to our EO, ranging from 2.1 to $4.0 \%$. However, there were also some differences; for example, $\alpha$-terpinyl acetate was present in EOs from Tuscany (Italy) and Australia, but it was totally absent in the EO studied [37,39] and dihydroeugenol, present in our sample, was absent in the EP EO from Morocco, Australia, and Tuscany [37-39]. An interesting study, which compared the chemistry and bioactivity of E. globulus EOs obtained by different extraction methods, concluded that there are some significant differences between EOs both in terms of high total yield and high concentration of volatiles [40]. Thus, the bioactivity of the EOs is also completely different and could be employed in different applications. This study can be a starting point for new research aimed at comparing different ways of obtaining EOs and selecting the most productive ones in the circular economy. In this way, it could be possible to implement these EO applications as bioherbicides, increasing the efficiency, soil persistency, and so on.

\subsection{Phytotoxic and Anti- $\alpha$-Amylase Activity}

The possible phytotoxic activities of our EOs were tested on both weed (Lolium multiflorum Lam., Portulaca oleracea L.) and crop (Raphanus sativus L., Lactuca sativa L., Lepidium sativum L., Solanum lycopersicum L., Pisum sativum L., Cucumis sativus L.) plant species, in order to investigate both the possible herbicidal activity against weeds and the possible negative impact on crop growth.

Tables 3 and 4 show the results of phytotoxic activity of the selected EOs against all seeds tested. 
Table 3. Inhibition of germination of L. sativa, P. oleracea, R.sativus, S. lycopersicum, P. sativum, C. sativus, L. sativum, and L. multiflorum seeds treated with different doses of EOs. Results are reported as the mean \pm SD of three experiments. ${ }^{*} p<0.05$, ${ }^{* *} p<0.01$, vs. control (inhibition $=0$ ), according to two way ANOVA followed by Tuckey's multiple comparison test, at the significance level of $p<0.05$.

\begin{tabular}{|c|c|c|c|c|c|c|c|c|c|}
\hline \multirow{2}{*}{\multicolumn{2}{|c|}{ Seeds }} & \multicolumn{4}{|c|}{ E. gunnii EO $(\mu \mathrm{g} / \mathrm{mL})$} & \multicolumn{4}{|c|}{ E. pulverulenta EO $(\mu \mathrm{g} / \mathrm{mL})$} \\
\hline & & \multirow{2}{*}{$\begin{array}{c}\mathbf{1 0 0 0} \\
7.7 \pm 0.6 \\
9.4\end{array}$} & \multirow{2}{*}{$\begin{array}{c}500 \\
8.3 \pm 0.6 \\
2.3\end{array}$} & \multirow{2}{*}{$\begin{array}{c}250 \\
8.0 \pm 0.0 \\
0\end{array}$} & \multirow{2}{*}{$\begin{array}{c}100 \\
9.5 \pm 0.7 \\
0\end{array}$} & \multirow{2}{*}{$\begin{array}{c}\mathbf{1 0 0 0} \\
9.0 \pm 0.0 \\
0\end{array}$} & \multirow{2}{*}{$\begin{array}{c}\mathbf{5 0 0} \\
8.0 \pm 0.0 \\
5.9\end{array}$} & \multirow{2}{*}{$\begin{array}{c}250 \\
8.5 \pm 0.7 \\
0\end{array}$} & \multirow{2}{*}{$\begin{array}{c}100 \\
8.5 \pm 0.7 \\
0\end{array}$} \\
\hline L. sativa & $\begin{array}{c}\text { Mean } \pm \text { SD } \\
\%\end{array}$ & & & & & & & & \\
\hline P. oleracea & $\begin{array}{c}\text { Mean } \pm \text { SD } \\
\%\end{array}$ & $\begin{array}{c}0.3 \pm 0.6 * \\
94.6\end{array}$ & $\begin{array}{c}2.3 \pm 2.3 \\
58.9\end{array}$ & $\begin{array}{c}2.7 \pm 2.1 \\
51.8\end{array}$ & $\begin{array}{c}3.3 \pm 2.1 \\
41.1\end{array}$ & $\begin{array}{c}1.7 \pm 1.5^{* *} \\
74.7\end{array}$ & $\begin{array}{c}2.0 \pm 1.7^{* *} \\
70.2\end{array}$ & $\begin{array}{c}4.3 \pm 1.5 \\
35.9\end{array}$ & $\begin{array}{c}2.7 \pm 1.2 * \\
59.8\end{array}$ \\
\hline R. satious & $\underset{\%}{\text { Mean } \pm \text { SD }}$ & $\begin{array}{c}2.7 \pm 0.6^{* *} \\
61.5\end{array}$ & $\begin{array}{c}3.7 \pm 0.6^{*} \\
47.2\end{array}$ & $\begin{array}{c}5.0 \pm 2.6 \\
28.6\end{array}$ & $\begin{array}{c}3.7 \pm 0.6^{*} \\
47.8\end{array}$ & $\begin{array}{c}4.0 \pm 0.0 \\
36.6\end{array}$ & $\begin{array}{c}3.7 \pm 1.2 * \\
41.3\end{array}$ & $\begin{array}{c}3.7 \pm 1.5^{*} \\
41.3\end{array}$ & $\begin{array}{c}6.0 \pm 1.7 \\
38.4\end{array}$ \\
\hline $\begin{array}{l}\text { S. lycoper- } \\
\text { sicum }\end{array}$ & $\underset{\%}{\operatorname{Mean} \pm \mathrm{SD}}$ & $\begin{array}{c}2.6 \pm 1.5^{* *} \\
67.5\end{array}$ & $\begin{array}{c}4.5 \pm 0.7^{*} \\
43.8\end{array}$ & $\begin{array}{c}5.7 \pm 0.6 \\
27.8\end{array}$ & $\begin{array}{c}5.6 \pm 0.6 \\
30\end{array}$ & $\begin{array}{c}8.3 \pm 0.6 \\
0\end{array}$ & $\begin{array}{c}7.3 \pm 0.6 \\
8.7\end{array}$ & $\begin{array}{c}7.3 \pm 0.6 \\
8.7\end{array}$ & $\begin{array}{c}8.2 \pm 0.6 \\
0\end{array}$ \\
\hline P. sativum & $\underset{\%}{\text { Mean } \pm \text { SD }}$ & $\begin{array}{c}9.0 \pm 1.7 \\
7.2\end{array}$ & $\begin{array}{c}9.0 \pm 1.7 \\
7.2\end{array}$ & $\begin{array}{c}7.3 \pm 2.1 \\
24.7\end{array}$ & $\begin{array}{c}9.3 \pm 1.2 \\
4.1\end{array}$ & $\begin{array}{c}9.3 \pm 0.6 \\
4.1\end{array}$ & $\begin{array}{c}8.7 \pm 0.6 \\
10.3\end{array}$ & $\begin{array}{c}10 \pm 0.0 \\
3\end{array}$ & $\begin{array}{c}9.7 \pm 0.6 \\
0\end{array}$ \\
\hline C. sativus & $\begin{array}{c}\text { Mean } \pm \text { SD } \\
\%\end{array}$ & $\begin{array}{c}6.0 \pm 0.0 \\
25\end{array}$ & $\begin{array}{c}5.3 \pm 0.6 \\
33.7\end{array}$ & $\begin{array}{c}5.7 \pm 0.6 \\
28.7\end{array}$ & $\begin{array}{c}4.3 \pm 0.6 \\
46.2\end{array}$ & $\begin{array}{c}5.3 \pm 0.6 \\
11.6\end{array}$ & $\begin{array}{c}5.7 \pm 0.6 \\
5\end{array}$ & $\begin{array}{c}6.0 \pm 0.0 \\
0\end{array}$ & $\begin{array}{c}6.3 \pm 0.6 \\
0\end{array}$ \\
\hline L. sativum & $\begin{array}{c}\text { Mean } \pm \text { SD } \\
\%\end{array}$ & $\begin{array}{c}9.0 \pm 1.0 \\
5.5\end{array}$ & $\begin{array}{c}8.3 \pm 0.6 \\
2.3\end{array}$ & $\begin{array}{c}7.5 \pm 0.7 \\
11.8\end{array}$ & $\begin{array}{c}9.1 \pm 0.3 \\
0\end{array}$ & $\begin{array}{c}7.0 \pm 0.0 \\
12.5\end{array}$ & $\begin{array}{c}6.7 \pm 1.5 \\
16.2\end{array}$ & $\begin{array}{c}6.7 \pm 2.1 \\
16.2\end{array}$ & $\begin{array}{c}8.0 \pm 1.7 \\
0\end{array}$ \\
\hline $\begin{array}{c}\text { L. } \\
\text { multiflorum }\end{array}$ & $\begin{array}{c}\text { Mean } \pm \text { SD } \\
\%\end{array}$ & $\begin{array}{c}7.0 \pm 2.0 \\
12.5\end{array}$ & $\begin{array}{c}7.5 \pm 0.7 \\
6.2\end{array}$ & $\begin{array}{c}7.7 \pm 0.6 \\
3.7\end{array}$ & $\begin{array}{c}7.0 \pm 2.6 \\
12.5\end{array}$ & $\begin{array}{c}7.3 \pm 0.6 \\
0\end{array}$ & $\begin{array}{c}6.0 \pm 1.0 \\
14.2\end{array}$ & $\begin{array}{c}7.0 \pm 0.0 \\
0\end{array}$ & $\begin{array}{c}8.3 \pm 0.6 \\
0\end{array}$ \\
\hline
\end{tabular}

Table 4. Inhibition of radical elongation of L. sativa, P. oleracea, R.sativus, S. lycopersicum, P. sativum, C. sativus, L. sativum, and L. multiflorum seeds treated with different doses. Results are reported as the mean \pm SD of three experiments. ${ }^{*} p<0.05$, ${ }^{* *} p<0.01$, vs. control (inhibition $=0$ ), according to two way ANOVA followed by Tuckey's multiple comparison test, at the significance level of $p<0.05$.

\begin{tabular}{|c|c|c|c|c|c|c|c|c|c|}
\hline \multirow{2}{*}{\multicolumn{2}{|c|}{ Seeds }} & \multicolumn{4}{|c|}{ E. gunnii EO $(\mu \mathrm{g} / \mathrm{mL})$} & \multicolumn{4}{|c|}{ E. pulverulenta EO $(\mu \mathrm{g} / \mathrm{mL})$} \\
\hline & & \multirow{2}{*}{$\begin{array}{c}1000 \\
1.7 \pm 0.6 \\
37.0\end{array}$} & \multirow{2}{*}{$\begin{array}{c}500 \\
2.5 \pm 0.5 \\
7.4\end{array}$} & \multirow{2}{*}{$\begin{array}{c}\mathbf{2 5 0} \\
2.3 \pm 0.6 \\
17\end{array}$} & \multirow{2}{*}{$\frac{100}{2.6 \pm 0.7} \frac{0.7}{3}$} & \multirow{2}{*}{$\begin{array}{c}1000 \\
2.2 \pm 0.5 \\
4.5\end{array}$} & \multirow{2}{*}{$\begin{array}{c}500 \\
2.1 \pm 0.3 \\
9.5\end{array}$} & \multirow{2}{*}{$\begin{array}{c}250 \\
2.2 \pm 0.5 \\
4.5\end{array}$} & \multirow{2}{*}{$\begin{array}{c}100 \\
2.1 \pm 0.5 \\
9.5\end{array}$} \\
\hline L. sativa & $\begin{array}{c}\text { Mean } \pm \text { SD } \\
\%\end{array}$ & & & & & & & & \\
\hline P. oleracea & $\begin{array}{c}\text { Mean } \pm \text { SD } \\
\%\end{array}$ & $\begin{array}{c}2.3 \pm 0.4 \\
0\end{array}$ & $\begin{array}{c}2.1 \pm 0.2 \\
0\end{array}$ & $\begin{array}{c}2.2 \pm 0.3 \\
0\end{array}$ & $\begin{array}{c}2.0 \pm 0.1 \\
0\end{array}$ & $\begin{array}{c}1.9 \pm 0.4 \\
0\end{array}$ & $\begin{array}{c}2.1 \pm 0.3 \\
0\end{array}$ & $\begin{array}{c}2.3 \pm 0.2 \\
0\end{array}$ & $\begin{array}{c}2.2 \pm 0.2 \\
0\end{array}$ \\
\hline R. sativus & $\begin{array}{c}\text { Mean } \pm \text { SD } \\
\%\end{array}$ & $\begin{array}{c}1.0 \pm 0.2 \\
0\end{array}$ & $\begin{array}{c}1.2 \pm 0.6 \\
0\end{array}$ & $\begin{array}{c}1.5 \pm 0.3 \\
0\end{array}$ & $\begin{array}{c}1.7 \pm 0.4 \\
0\end{array}$ & $\begin{array}{c}1.8 \pm 0.5 \\
0\end{array}$ & $\begin{array}{c}1.5 \pm 0.8 \\
0\end{array}$ & $\begin{array}{c}1.7 \pm 0.3 \\
0\end{array}$ & $\begin{array}{c}2.4 \pm 0.6 \\
0\end{array}$ \\
\hline $\begin{array}{l}\text { S. lycoper- } \\
\text { sicum }\end{array}$ & $\begin{array}{c}\text { Mean } \pm \text { SD } \\
\%\end{array}$ & $\begin{array}{c}1.3 \pm 0.5^{* *} \\
82.6\end{array}$ & $\begin{array}{c}2.2 \pm 1.9 * \\
70.7\end{array}$ & $\begin{array}{c}2.3 \pm 1.7 \text { * } \\
69.3\end{array}$ & $\begin{array}{c}2.5 \pm 1.8^{*} \\
66.7\end{array}$ & $\begin{array}{c}3.2 \pm 1 \\
57.3\end{array}$ & $\begin{array}{c}4.5 \pm 1.7 \\
40\end{array}$ & $\begin{array}{c}4.6 \pm 1.7 \\
38.7\end{array}$ & $\begin{array}{c}5.8 \pm 1.8 \\
22.7\end{array}$ \\
\hline P. sativum & $\underset{\%}{\text { Mean } \pm \text { SD }}$ & $\begin{array}{c}4.1 \pm 1.0 \\
0\end{array}$ & $\begin{array}{c}4.0 \pm 1.0 \\
0\end{array}$ & $\begin{array}{c}4.5 \pm 1.1 \\
0\end{array}$ & $\begin{array}{c}3.9 \pm 0.9 \\
0\end{array}$ & $\begin{array}{c}3.9 \pm 0.6 \\
0\end{array}$ & $\begin{array}{c}3.7 \pm 0.8 \\
0\end{array}$ & $\begin{array}{c}4.5 \pm 1.1 \\
0\end{array}$ & $\begin{array}{c}4.8 \pm 0.9 \\
0\end{array}$ \\
\hline C. sativus & $\begin{array}{c}\text { Mean } \pm \text { SD } \\
\%\end{array}$ & $\begin{array}{c}6.0 \pm 1.6 \\
15.5\end{array}$ & $\begin{array}{c}7.0 \pm 1.0 \\
1.4\end{array}$ & $\begin{array}{c}6.9 \pm 1.7 \\
2.8\end{array}$ & $\begin{array}{c}6.4 \pm 0.8 \\
9.9\end{array}$ & $\begin{array}{c}5.4 \pm 0.7 \\
23.9\end{array}$ & $\begin{array}{c}7.5 \pm 0.9 \\
0\end{array}$ & $\begin{array}{c}6.7 \pm 1.1 \\
5.6\end{array}$ & $\begin{array}{c}7.2 \pm 1.9 \\
0\end{array}$ \\
\hline L. sativum & $\begin{array}{c}\text { Mean } \pm \text { SD } \\
\%\end{array}$ & $\begin{array}{c}2.0 \pm 1.4 \\
63.0\end{array}$ & $\begin{array}{c}3.0 \pm 1.2 \\
44.4\end{array}$ & $\begin{array}{c}3.2 \pm 1.3 \\
40.7\end{array}$ & $\begin{array}{c}2.5 \pm 1.8 \\
53.7\end{array}$ & $\begin{array}{c}1.1 \pm 0.2 * * \\
76.0\end{array}$ & $\begin{array}{c}1.9 \pm 0.4 * \\
58.7\end{array}$ & $\begin{array}{c}2.2 \pm 0.8^{*} \\
52.2\end{array}$ & $\begin{array}{c}2.5 \pm 0.7^{*} \\
45.6\end{array}$ \\
\hline $\begin{array}{c}\text { L. } \\
\text { multiflorum }\end{array}$ & $\underset{\%}{\text { Mean } \pm \text { SD }}$ & $\begin{array}{c}2.6 \pm 0.6^{*} \\
53.5\end{array}$ & $\begin{array}{c}3.5 \pm 1.1 \\
37.5\end{array}$ & $\begin{array}{c}4.0 \pm 1.0 \\
28.6\end{array}$ & $\begin{array}{c}3.5 \pm 1.0 \\
37.5\end{array}$ & $\begin{array}{c}1.6 \pm 0.7^{* *} \\
70.4\end{array}$ & $\begin{array}{c}1.7 \pm 0.4^{* *} \\
68.5\end{array}$ & $\begin{array}{c}2.3 \pm 0.7^{* *} \\
57.4\end{array}$ & $\begin{array}{c}3.4 \pm 0.7 \\
37.0\end{array}$ \\
\hline
\end{tabular}

EG EO was able to inhibit the germination of P. oleracea, R. sativus, and S. lycopersicum seeds, while affecting only radical elongation in S. lycopersicum seeds (Figure 7). 
E. gunnii

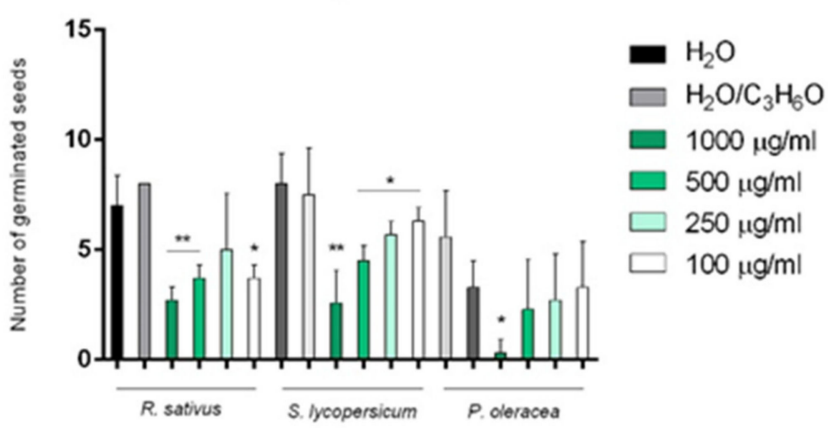

E. gunnii

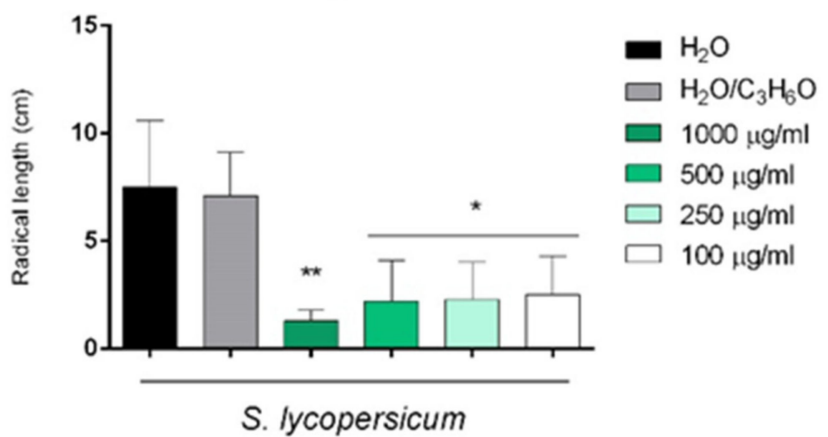

Figure 7. Phytotoxic activity of E. gunnii (EG) EO against the germination of P. oleracea, R. sativus, and S. lycopersicum and the radical elongation of $S$. lycopersicum $120 \mathrm{~h}$ after sowing. The results are the mean of three experiments \pm standard deviation. ${ }^{*} p<0.05$ and ${ }^{* *} p<0.01$ compared with control (ANOVA followed by Dunnett's multiple comparison test).

Instead, EP EO inhibited the germination of P. oleracea and R. sativus, and affected the radical elongation of L. sativum and L. multiflorum (Figure 8).

\section{E. pulverulenta}

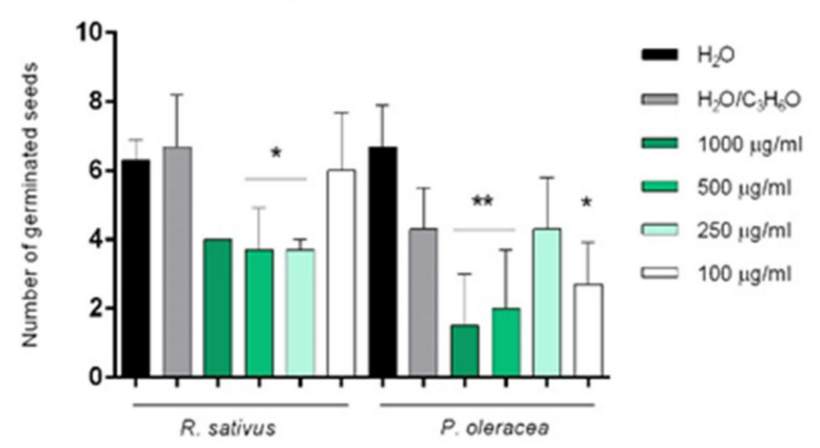

E. pulverulenta

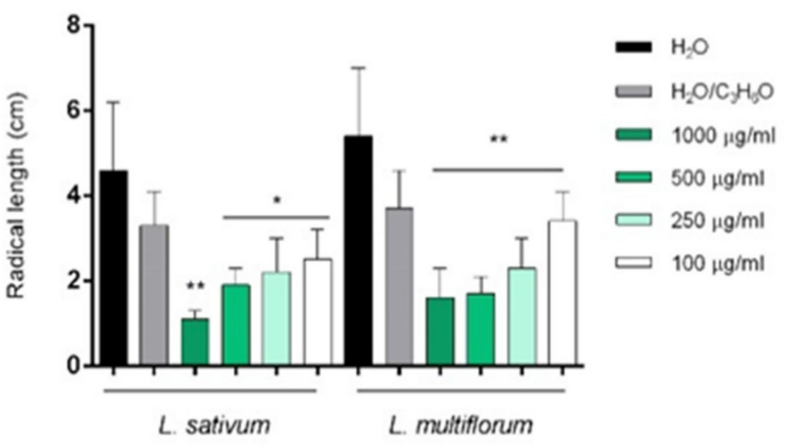

Figure 8. Phytotoxic activity of E. pulverulenta 'Baby blue' (EP) EO against germination of P. oleracea and R. sativus, and radical elongation of L. sativum and L. multiflorum $120 \mathrm{~h}$ after sowing. The results are the mean of three experiments \pm standard deviation. ${ }^{*} p<0.05$ and ${ }^{* *} p<0.01$ compared with control (ANOVA followed by Dunnett's multiple comparison test).

Moreover, the investigated EOs did not show any effects against the germination and/or radical elongation of L. sativa, P. sativum, and C. sativus.

Different species of Eucalyptus genus were studied for their allelopathic properties both against weeds and crops: E. citriodora Hook. was phytotoxic to Bidens pilosa L., Amaranthus viridis L., Rumex nepalensis Spreng., and Leucaena leucocephala (Lam.) de Wit, and caused injuries to Triticum aestivum L., Zea mays L., Raphanus sativus L., and Oryza sativa L. [23]; the EO from E. nicholii Maiden and Blakely strongly inhibited the germination of Amaranthus retroflexus L., Portulaca oleracea L., Acroptilon repens (L.) DC. [41], and E. tereticornis $\mathrm{Sm}$. EO possessed allelopathic potential against $A$. viridis [42]. Rasaeifar and coworkers [16] reported that essential oil of E. globulus Labill. had poisonous effects on important weeds, such as Amaranthus blitoides S.Watson and Cynodon dactylon L., suggesting further research into the involved mechanisms to allow its use in cultivated lands. A lemon-scented Eucalyptus showed high phytotoxicity and its use as a bioherbicide has been proposed [22]. Eucalyptus EOs have been studied for the control of the noxious weed, Parthenium hystorophorus L. [43]. E. globulus EO, with a high content of 1,8-cineole, significantly affected seedling growth, showing a post-emergent herbicidal activity in Echinochloa crus-galli (L.) P. Beauv. [44]. The volatile cineoles are recognized as allelopathic agents [45].

Another common terpenoid present in EOs from many Eucalyptus species, $\alpha$-pinene, played a key-role in root cell membranes' disruption [24,25]. Even eugenol possessed a 
weed-suppressing ability, negatively affecting the photosynthetic efficiency and the energy metabolism of different weed species [19]. Moreover, monoterpenes have been proposed for their pivotal role in Eucalyptus communities [46].

Even though volatile allelochemicals derived from Eucalyptus EOs are probably among the most investigated for their herbicidal properties, no studies are currently available on the possible phytotoxic activity of EP EO. Regarding EG, our previous study on EO from Sardinia showed no phytotoxic activity against several tested seeds [35].

$\alpha$-Amylase is a key enzyme involved in seed growth regulation; in fact, it hydrolyses the starch during the seed germination process [47]. Consequently, after the determination of the phytotoxic activity of the EOs, their possible activity on $\alpha$-amylase regulation was studied. The results reported in Table 5 showed that the EP EO, with an $\mathrm{IC}_{50}$ value of $35.9 \mathrm{\mu g} / \mathrm{mL}$, was more active than EG EO and acarbose, used as positive control.

Table 5. $\alpha$-Amylase inhibitory activity of E.gunnii and E. pulverulenta 'Baby blue' EOs.

\begin{tabular}{cc}
\hline Essential Oil & IC $_{\mathbf{5 0}}(\boldsymbol{\mu g} / \mathbf{m L})$ \\
\hline E. pulverulenta & $35.9 \pm 3.6$ \\
E. gunnii & $524.1 \pm 10.3$ \\
Acarbose (positive control) & $130.2 \pm 12.4$ \\
\hline
\end{tabular}

Data are expressed as mean \pm S.D. $(n=3)$.

In light of these results, it is possible to hypothesize that the variability in EOs phytotoxic activities may be related to the difference in the starch reserve of the seeds of the considered species. No previous studies reported the activity of EOs from Eucalyptus sp. pl. on $\alpha$-amylase in association with phytotoxic activity.

\subsection{Toxicity}

The eco-compatibility of EG and EP EOs was evaluated using the toxicological test on Artemia salina, a marine zooplanktonic organism, which represents the gold standard in toxicological assays, owing to its easy cultivation, availability, low cost, and adaptation to adverse conditions [48]. Both EOs were tested in a wide dose range $(0.01-100 \mathrm{mg} / \mathrm{mL})$.

Potassium bichromate, used as positive control, inhibited the nauplii vitality after 24 and 48 h by 90 and $98 \%$, respectively, showing a strong toxicity. The tested EOs did not show any toxicity or alteration of the nauplii swimming behaviour at 24 and $48 \mathrm{~h}$ until $10 \mathrm{mg} / \mathrm{mL}$, with any statistically significant differences with respect to the negative control (data not shown). On the contrary, a mild toxicity at $24 \mathrm{~h}(16.66 \mathrm{vs} .13 .33 \%$ for EG and EP EOs, respectively), which became strong at $48 \mathrm{~h}(85.0 \mathrm{vs} .50 \%$ for EG and EP EOs, respectively), was observed at the highest dose $(100 \mathrm{mg} / \mathrm{mL})$ for both EOs, with a statistically significant difference between them $(p<0.001$, data not shown).

This is the first study to investigate the toxicity of EG and EP EOs on Artemia salina and no data are currently available on the eco-compatibility of these EOs.

However, already from these preliminary data, it is possible to speculate that both Eucalyptus EOs tested are safe in a wide dose range $(0.01-10 \mathrm{mg} / \mathrm{mL})$ for aquatic organisms and that they could be used safely in agriculture as eco-sustainable bio-herbicides.

\section{Materials and Methods}

\subsection{Standards and Reagents}

Soluble starch, PPA (porcine pancreatic $\alpha$-amylase), $\mathrm{HCl}, \mathrm{KI}, \mathrm{I}_{2}, \mathrm{~K}_{2} \mathrm{Cr}_{2} \mathrm{O}_{7}$, acetone, and acarbose were purchased from Sigma Aldrich (Milan, Italy).

\subsection{Plant Material}

E. gunnii (EG) and E. pulverulenta 'Baby blue' (EP) were collected in January 2021 in commercial plantations in the hinterland of Western Liguria (Finale Ligure, Savona, Italy) in Tovo San Giacomo (coordinates $=44.19683185^{\circ} \mathrm{N}, 8.26007775^{\circ} \mathrm{E}$ ) and in Magliolo (coordinates $=44.1972954^{\circ} \mathrm{N}, 8.2558821^{\circ} \mathrm{E}$ ), respectively. A voucher specimen of each species 
(GE5187 and GE5188, respectively) was deposited in the herbarium of the Department of Earth, Environment and Life Sciences, University of Genoa, Italy. The plants were approximately 10 months old and about $3 \mathrm{~m}$ high at the time of collection. About $1 \mathrm{~kg}$ of branches bearing juvenile foliage of each species was collected for both micromorphological analyses and EOs' extraction.

\subsection{Extraction of Essential Oils}

The branches bearing juvenile foliage were reduced to small pieces and then subjected to hydrodistillation for $3 \mathrm{~h}$, according to the standard procedure described in the European Pharmacopoeia [49]. The hydrodistillation of the aerial parts of EG and EP furnished pale yellow EOs at a yield of 1.1 and $1.6 \%$ on a dry mass basis, respectively. The EOs were dried over anhydrous sodium sulphate and kept under $\mathrm{N}_{2}$ at $4{ }^{\circ} \mathrm{C}$ in the dark, until analysis.

\subsection{Micromorfological Analyses}

For each species, eight leaves were selected in sequence along a branch, starting from the first fully expanded juvenile leaf, for micromorphological analyses. Two leaves for species were used to obtain epidermal peelings using the nail polish technique [50]. Six images of the imprints were captured at $10 \times$ magnification, both for the abaxial and adaxial epidermal surfaces, avoiding the central rib. The observations were performed in fields with the area corresponding to $0.930 \mathrm{~mm}^{2}$ using a Leica D.M. 2000 microscope equipped with a digital camera (DFC 320, Leica Microsystems, Wetzlar, Germany). The oil gland density was obtained according to the overlying cells on the epidermal surfaces, in agreement with the method previously described by Santos and coworkers [28]. For the histochemical analysis, another three leaves of each species were fixed, dehydrated, and paraffin embedded. Eight-micron thick cross sections were obtained using an automatic advance rotative microtome (Leica RM 2255, Leica Biosystems, Heidelberg, Germany) and stained with Toluidine Blue $\mathrm{pH} 4.0$ [51] for anatomical and histological characterization. Microphotographs were taken using a Leica DMRB light microscope with a Leica CCD camera DFC420C (Leica, Switzerland).

Other leaf samples of both species were also processed for SEM analyses. Three portions for each species (about 1.5-2.0 $\mathrm{cm}^{2}$ ) were fixed in $70 \%$ ethanol-FineFix working solution (Milestone s.r.l., Bergamo, Italy) for $24 \mathrm{~h}$ at $4{ }^{\circ} \mathrm{C}$, dehydrated through ethanol series [52], and then critical point dried in $\mathrm{CO}_{2}$ (CPD, K850 2M Strumenti s.r.l., Rome, Italy). Finally, the samples were mounted on the aluminium stubs using glued carbon tabs, sputter-coated with $10 \mathrm{~nm}$ gold [53], and observed with a Vega3 Tescan LMU SEM (Tescan USA Inc., Cranberry Twp, PA, USA) at an accelerating voltage of $20 \mathrm{kV}$. For each species, six images for both abaxial and adaxial surfaces were captured at $200 \times$ magnification to determine oil gland density.

All the captured images were analysed using image processing software ImageJ [54], which enables measurements and counting, allowing to obtain quantitative data.

\subsection{GC-FID Analysis}

Analytical gas chromatography (GC) was carried out on a Perkin-Elmer Sigma-115 gas chromatograph (Perkin Elmer, Waltham, MA, USA) equipped with a flame ionization detector (FID) and a data handling processor. The separation was achieved using a HP-5 MS fused-silica capillary column $(30 \mathrm{~m} \times 0.25 \mathrm{~mm}$ i.d., $0.25 \mu \mathrm{m}$ film thickness, Agilent, Roma, Italy). Column temperature: $40{ }^{\circ} \mathrm{C}$, with $5 \mathrm{~min}$ initial hold, and then to $270{ }^{\circ} \mathrm{C}$ at $2{ }^{\circ} \mathrm{C} / \mathrm{min}$, $270^{\circ} \mathrm{C}(20 \mathrm{~min})$; injection mode, splitless $(1 \mu \mathrm{L}$ of a 1:1000 $n$-hexane solution). Injector and detector temperatures were $250{ }^{\circ} \mathrm{C}$ and $290^{\circ} \mathrm{C}$, respectively. The analysis was also run using a fused silica HP Innowax polyethylene glycol capillary column $(50 \mathrm{~m} \times 0.20 \mathrm{~mm}$ i.d., $0.25 \mu \mathrm{m}$ film thickness, Agilent, Roma, Italy). In both cases, helium was used as the carrier gas $(1.0 \mathrm{~mL} / \mathrm{min})$. 


\subsection{GC/MS Analysis}

Analysis was performed on an Agilent 6850 Ser. II apparatus (Agilent, Roma, Italy), fitted with a fused silica DB- 5 capillary column $(30 \mathrm{~m} \times 0.25 \mathrm{~mm}$ i.d., $0.33 \mu \mathrm{m}$ film thickness, Agilent, Roma, Italy), coupled to an Agilent Mass Selective Detector MSD 5973; ionization energy voltage $70 \mathrm{eV}$; electron multiplier voltage energy $2000 \mathrm{~V}$. Mass spectra (MS) were scanned in the range 40-500 amu, scan time 5 scans/s. Gas chromatographic conditions were as reported in the previous paragraph; transfer line temperature, $295^{\circ} \mathrm{C}$.

\subsection{Identification of the Essential Oil Components}

Most constituents were identified by GC by comparison of their Kovats retention indices (Ri) (determined relative to the retention times (tR) of $n$-alkanes $\left(C_{10}-C_{35}\right)$ ), with either those of the literature [55-58] and mass spectra on both columns or those of authentic compounds available in our laboratories by means of NIST 02 and Wiley 275 libraries [59]. The component relative concentrations were obtained by peak area normalization. No response factors were calculated.

\subsection{Phytotoxic Activity}

The phytotoxic activity was evaluated on germination and radical elongation of several weed and crop species: Raphanus sativus L., Lactuca sativa L., Lepidium sativum L., Solanum lycopersicum L., Pisum sativum L., Cucumis sativus L., Lolium multiflorum Lam., and Portulaca oleracea $\mathrm{L}$. These seeds are often used for their easy and well-known germinability. $R$. sativus, L. sativa, L. sativum, S. lycopersicum, P. sativum, and C. sativus seeds were purchased from Blumen group SRL (Emilia-Romagna, Bologna, Italy). L. multiflorum seeds were purchased from Fratelli Ingegnoli Spa (Milano, Italy), and P. oleracea seeds from W. Legutko SRL (Jutrosin, Poland). The seeds were sterilized in $95 \%$ ethanol for $15 \mathrm{~s}$ and sown in Petri dishes $(\varnothing=90 \mathrm{~mm})$, on three layers of Whatman filter paper. They were impregnated with $7 \mathrm{~mL}$ of deionized water used as first control to verify the germinability of the seeds, $7 \mathrm{~mL}$ of a water-acetone mixture (99.5:0.5, $v / v)$ as second control because EOs were dissolved in this mixture for their lipophilicity, or $7 \mathrm{~mL}$ of the tested solution at different doses $(1000,500,250$, and $100 \mathrm{mg} / \mathrm{mL})$. Controls, carried out with the water-acetone mixture alone, showed no appreciable differences in comparison with control in water alone. The germination conditions were $20 \pm 1{ }^{\circ} \mathrm{C}$, with a natural photoperiod. Seed germination was observed in Petri dishes every $24 \mathrm{~h}$. A seed was considered germinated when the protrusion of the root became evident [60]. On the fifth day (after $120 \mathrm{~h}$ ) for $R$. sativus and on the tenth day (after $240 \mathrm{~h}$ ) for the other tested seeds, the effects on radicle elongation were measured in $\mathrm{cm}$. Each determination was repeated three times, using Petri dishes containing 10 seeds each. Data are expressed as the mean \pm standard deviation for both germination and radicle elongation.

\section{9. $\alpha$-Amylase Inhibitory Assay}

Screening of plant material for $\alpha$-amylase inhibition was carried out according to Xiao and coworkers [61] and Sudha and coworkers [62], based on the starch-iodine test with some modification. The total assay mixture composed of $200 \mu \mathrm{L} 0.02 \mathrm{M}$ sodium phosphate buffer $\mathrm{pH} 6.9$ containing 2 units $/ \mathrm{mL}$ of PPA, and EOs at a dose from 0.01 to $1.0 \mathrm{mg} / \mathrm{mL}$ $(w / v)$ were incubated at $37^{\circ} \mathrm{C}$ for $10 \mathrm{~min}$. Then, soluble starch $(0.5 \%, w / v)$ was added to each reaction cuvette and incubated at $37^{\circ} \mathrm{C}$ for $15 \mathrm{~min}$. Then, $1 \mathrm{M} \mathrm{HCl}(100 \mu \mathrm{L})$ was added to stop the enzymatic reaction, followed by the addition of $400 \mu \mathrm{L}$ of iodine reagent ( $5 \mathrm{mM} \mathrm{I}_{2}$ and $5 \mathrm{mM} \mathrm{KI}$ ). The colour change was recorded, and the absorbance was read at $620 \mathrm{~nm}$ on a microplate reader. The control reaction representing $100 \%$ enzyme activity did not contain any EO. The known PPA inhibitor, acarbose, was used as a positive control at a concentration range of $0.01-1 \mathrm{mg} / \mathrm{mL}$. Finally, the $\alpha$-amylase inhibition activity was calculated with the following formula: 


$$
\alpha-\text { amylase inhibition activity }(\%)=\frac{A b s_{1}}{A b s_{2}} 100
$$

where $A b s_{1}$ is the absorbance of sample solution mixed with amylase solution and soluble starch solution, and $A b_{2}$ is the absorbance of the control (sample solution mixed with soluble starch solution). The $\mathrm{IC}_{50}$ values were defined as the dose of the $\mathrm{EO}$, containing the $\alpha$-amylase inhibitor, that inhibited $50 \%$ of the PPA activity.

\subsection{Brine Shrimp Lethality Assay}

In order to investigate the toxicity of the EG and EP EOs, the brine shrimp (Artemia salina) lethality assay was carried out according to Caputo and coworkers [35]. Brine shrimp's eggs were purchased by a fish shop, placed in a hatcher chamber containing seawater, and incubated for $48 \mathrm{~h}$ at room temperature with continuous aeration and illumination. Stock solutions $(0.01-100 \mathrm{~g} / \mathrm{mL}$ ) of EG and EP EOs, as well as potassium bichromate $\left(\mathrm{K}_{2} \mathrm{Cr}_{2} \mathrm{O}_{7}, 50 \mathrm{mg} / \mathrm{mL}\right)$ used as a positive control, were prepared in DMSO and water, respectively. After that, $2 \mu \mathrm{L}$ of each sample and control were seeded in a 24-well plate, and diluted 1:1000 $v / v$ in seawater in order to achieve a $0.1 \%$ DMSO non-toxic concentration. Ten nauplii per well were added and incubated for $48 \mathrm{~h}$ under the same conditions reported above. Surviving larvae without abnormal swimming behavior after $24 \mathrm{~h}$ and $48 \mathrm{~h}$ were counted by a stereomicroscope (SMZ-171 Series, Motic Asia, Hong Kong, China). A negative control (ten larvae treated with $0.1 \%$ DMSO in seawater) was evaluated. Three independent experiments $(n=10)$ were carried out for each treatment. Lethality was calculated using the following equation:

$$
\% \text { Lethality }=100-[(\text { slt } \times 100)] / \text { slcs }
$$

where slt are the survival larvae treated with the extracts or $\mathrm{K}_{2} \mathrm{Cr}_{2} \mathrm{O}_{7}$, and slcs are the survival larvae treated with seawater (negative control).

\section{Conclusions}

Our study investigated the micromorphology, phytochemistry, phytotoxic activity, and eco-compatibility of E. gunnii (EG) and E. pulverulenta 'Baby blue' (EP) EOs. The comparison between the two species showed that EP had a higher oil gland density as well as a higher yield in EO with respect to EG. In both EOs, 1.8-cineole was the major compound $(\sim 75 \%) ; \alpha$-pinene was the second most abundant component in EG, while eugenol was the second in EP. Several studies have shown the phytotoxic activities of the EOs rich in terpenes, often linked to a high concentration of the afore-mentioned compounds. Our research highlighted the potential phytotoxicity of the EG and EP EOs against several weeds: both EOs inhibited Portulaca oleracea seed germination and EP EO also inhibited Lolium multiflorum radical elongation. Concerning crop species, the EOs investigated showed no effect against germination and/or radical growing of Lactuca sativa, Pisum sativum, and Cucumis sativus. On the contrary, EOs tested showed phytotoxicity on Solanum lycopersicum, Lepidium sativum, and Raphanus sativus. Our study also attested the inhibitory effects on a key enzyme involved in seed growth regulation, $\alpha$-amylase; EP EO was much more active than EG EO.

The data collected show that the allelopathic action mechanisms of these EOs are various and they can be used as selective bioherbicides on target species. In addition, the high eco-compatibility in a wide dose range of the two EOs studied suggests their possible safe applications in agriculture. Finally, we point out the potential of remnants from Eucalyptus pruning for their transformation into value-added products for sustainable agriculture programs. In fact, weed control with allelopathic compounds released from crop residues is today regarded as a safe strategy in crop management systems in the frame of the circular economy, and by-products rich in essential oils play a primary role in this context. 
Author Contributions: Conceptualization, V.D.F., L.C. (Laura Cornara), and D.T; data curation, L.C. (Lucia Caputo), L.C. (Laura Cornara), C.D., A.S., G.A., and L.D.M., formal analysis, L.C. (Lucia Caputo), L.C. (Laura Cornara), C.D.,V.D.F., G.A., D.T., and A.S.; investigation, L.C. (Lucia Caputo), L.C. (Laura Cornara), C.D., V.D.F., G.A., D.T., and A.S.; methodology, L.C. (Laura Cornara), V.D.F., D.T., and A.S.; resources, P.A.; validation, L.C. (Laura Cornara), L.C. (Lucia Caputo), V.D.F., D.T., and A.S.; writing-original draft, L.C. (Lucia Caputo), L.C. (Laura Cornara), C.D.,V.D.F., C.D., D.T., and A.S.; writing-review and editing, L.C. (Lucia Caputo), L.C. (Laura Cornara), C.D., L.D.M., V.D.F., D.T., and A.S. All authors have read and agreed to the published version of the manuscript.

Funding: This research received no external funding.

Institutional Review Board Statement: Not applicable.

Informed Consent Statement: Not applicable.

Data Availability Statement: The data presented in this study are available on request from the corresponding author.

Acknowledgments: We are very grateful to Luca Mastracci (DISC, University of Genoa) for his help in the preparation of histological samples.

Conflicts of Interest: The authors declare no conflict of interest.

Sample Availability: Samples of the E. gunnii and E. pulverulenta 'Baby blue' essential oils are available from the authors. Vouchers were deposited in the Herbarium of Department of Earth, Environment and Life Sciences, University of Genoa.

\section{References}

1. Dhakad, A.K.; Pandey, V.V.; Beg, S.; Rawat, J.M.; Singh, A. Biological, medicinal and toxicological significance of Eucalyptus leaf essential oil: A review. J. Sci. Food Agric. 2018, 98, 833-848. [CrossRef] [PubMed]

2. Tomé, M.; Almeida, M.H.; Barreiro, S.; Branco, M.; Deus, E.; Pinto, G.; Silva, J.; Soares, P.; Rodríguez-Soalleir, R. Opportunities and challenges of Eucalyptus plantations in Europe: The Iberian Peninsula experience. Eur. J. For. Res. 2021, 140, 489-510. [CrossRef]

3. Floris, I.; Cocco, A.; Buffa, F.; Mannu, R.; Satta, A. Insect pests of Eucalyptus plantations in Sardinia (Italy). J. Zool. 2018, 101, 61-71. [CrossRef]

4. Campisi, E.; Mancianti, F.; Pini, G.; Faggi, E.; Gargani, G. Investigation in central Italy of the possible association between Cryptococcus neoformans var. Gattii and Eucalyptus camaldulensis. Eur. J. Epidemiol. 2003, 18, 357-362. [CrossRef]

5. Forrest, M. The Performance of a Eucalyptus Gunnii Cut Foliage Plantation over 7 Years. Irish J. Agric. Food Res. 2002, 41, 235-245. Available online: http:/ / www.jstor.org/stable/25562467 (accessed on 4 October 2021).

6. Pacifici, S.; Ferrante, A.; Mensuali, A.; Serra, G. Postharvest physiology and technology of cut Eucalyptus branches: A review. Agr. Med. 2007, 137, 124-131.

7. Wirthensohn, M.G.; Collins, G.; Jones, G.P.; Sedgley, M. Variability in waxiness of Eucalyptus gunnii foliage for floriculture. Sci. Hortic. 1999, 82, 279-288. [CrossRef]

8. Garcia, C.; Montero, G.; Coronado, O.M.; Valdez Salaz, B.; Stoytcheva, M.; Rosas, N.; Ricardo, T.; Sagaste, C. Valorization of Eucalyptus Leaves by Essential Oil Extraction as an Added Value Product in Mexico. Waste Biomass Valor. 2017, 8, 1187-1197. [CrossRef]

9. Mediavilla, I.; Guillamón, E.; Ruiz, A.; Estepan, L.S. Essential Oils from Residual Foliage of Forest Tree and Shrub Species: Yield and Antioxidant Capacity. Molecules 2021, 26, 3257. [CrossRef] [PubMed]

10. Smeriglio, A.; Trombetta, D.; Cornara, L.; Valussi, M.; De Feo, V.; Caputo, L. Characterization and Phytotoxicity Assessment of Essential Oils from Plant Byproducts. Molecules 2019, 24, 2941. [CrossRef] [PubMed]

11. Lemke, N.; Murawski, A.; Schmied-Tobies, M.I.H.; Rucic, E.; Hoppe, H.W.; Conrad, A.; Kolossa-Gehring, M. Glyphosate and aminomethylphosphonic acid (AMPA) in urine of children and adolescents in Germany-Human biomonitoring results of the German Environmental Survey 2014-2017 (GerES V). Environ. Int. 2021, 156, 106769. [CrossRef]

12. Meftaul, I.M.; Venkateswarlu, K.; Dharmarajan, R.; Annamalai, P.; Asaduzzaman, M.; Parven, A.; Megharaj, M. Controversies over human health and ecological impacts of glyphosate: Is it to be banned in modern agriculture? Environ. Pollut. 2020, 263, 114372. Available online: https://www.sciencedirect.com/science/article/pii/S0269749119368265 (accessed on 4 October 2021). [CrossRef]

13. Setia, N.; Batish, D.R.; Singh, H.P.; Kohli, R.K. Phytotoxicity of volatile oil from Eucalyptus citriodora against some weedy species. J. Environ. Biol. 2007, 28, 63-66.

14. Kordali, S.; Cakir, A.; Sutay, S. Inhibitory Effects of Monoterpenes on Seed Germination and Seedling Growth. J. Biosci. 2007, 62, 207-214. [CrossRef] [PubMed]

15. Batish, D.; Singh, H.P.; Kohli, R.; Kaur, S. Eucalyptus essential oil as natural pesticide. For. Ecol. Manag. 2008, $256,2166-2174$. [CrossRef] 
16. Rasaeifar, M.; Hosseini, N.; Asl, N.; Zandi, P.; Aghdam, A. Allelopathic effect of Eucalyptus globulus' essential oil on seed germination and seedling establishment of Amaranthus blitoides and Cyndon dactylon. Trakia J. Sci. 2013, 11, 73-81.

17. Kanatas, P. Potential role of Eucalyptus spp. and Acacia spp. allelochemicals in weed management. Chil. J. Agric. Res. 2020, 80, 452-458. [CrossRef]

18. Puig, C.; Revilla, P.; Barreal, M.E.; Reigosa, R.M.; Pedrol, N. On the suitability of Eucalyptus globulus green manure for field weed control. Crop Prot. 2019, 121, 57-65. [CrossRef]

19. Vaid, S.; Batish, D.; Singh, D.H.; Kohli, R. Phytotoxic effect of eugenol towards two weedy species. Bioscan 2010, 5, 339-341.

20. Ibáñez, M.D.; Blázquez, M.A. Phytotoxic Effects of Commercial Eucalyptus citriodora, Lavandula angustifolia, and Pinus sylvestris Essential Oils on Weeds, Crops, and Invasive Species. Molecules 2019, 24, 2847. [CrossRef]

21. Verdeguer, M.; Blázquez, M.; Boira, H. Phytotoxic effects of Lantana camara, Eucalyptus camaldulensis and Eriocephalus africanus essential oils in weeds of Mediterranean summer crops. Biochem. Syst. Ecol. 2009, 37, 362-369. [CrossRef]

22. Batish, D.R.; Setia, N.; Singh, H.P.; Kohli, R.K. Phytotoxicity of lemon-scented Eucalypt oil and its potential use as a bioherbicide. Crop Prot. 2004, 23, 1209-1214. [CrossRef]

23. Batish, D.R.; Singh, H.P.; Nidhi, S.; Shalinder, K.; Kohli, R.K. Chemical composition and inhibitory activity of essential oil from decaying leaves of Eucalyptus citriodora. Z. Naturforsch. 2006, 61, 52-56. [CrossRef]

24. Singh, H.P.; Batish, D.; Kaur, S.; Arora, K.; Kohli, R. $\alpha$-Pinene Inhibits Growth and Induces Oxidative Stress in Roots. Ann. Bot. 2007, 98, 1261-1269. [CrossRef]

25. Zhang, J.; An, M.; Wu, H.; Stanton, R.; Lemerle, D. Chemistry and bioactivity of Eucalyptus essential oils. Allelopath. J. 2010, 25, 313-330.

26. WHO. Quality Control Methods for Herbal Materials; WHO Press: Valletta, Malta, 2011; Available online: https: / apps.who.int/ iris/handle/10665/44479 (accessed on 4 October 2021).

27. Alamgir, A.N.M. Microscopy in Pharmacognosy. In Therapeutic Use of Medicinal Plants and Their Extracts: Volume 1. Progress in Drug Research; Springer: Cham, Swizerland, 2017; Volume 73, Available online: https:/ /doi.org/10.1007/978-3-319-63862-1_11 (accessed on 4 October 2021). [CrossRef]

28. Santos, L.; Thadeo, M.; Iarema, L.; Meira, R.M.; Ferreira, F. Foliar anatomy and histochemistry in seven species of Eucalyptus. Rev. Arvore 2008, 32, 769-779. [CrossRef]

29. Migacz, I.; Raman, V.; Nisgoski, S.; Muniz, G.; Manfron, J.; Farago, P.; Khan, I.; Raeski, P. Comparative leaf morpho-anatomy of six species of Eucalyptus cultivated in Brazil. Rev. Bras. Farmacogn. 2018, 28, 273-281. [CrossRef]

30. Soliman, F.; Fathy, M.; Salama, M.; Saber, F. Botanical study of Eucalyptus cinerea F. Muell. ex Benth. Cultivated in Egypt. World J. Pharm. Pharmaceut. Sci. 2015, 4, 310-328.

31. King, D.; Gleadow, R.; Woodrow, I. Regulation of oil accumulation in single glands of Eucalyptus polybractea. New Phytol. 2006, 172, 440-451. [CrossRef] [PubMed]

32. Brooker, M.I.H.; Nicolle, D. Atlas of Leaf Venation and Oil Gland Patterns in the Eucalypts; Csiro Publishing: Collingwood, Australia, 2013.

33. Lucia, A.; Licastro, S.; Zerba, E.; Masuh, H. Yield, chemical composition, and bioactivity of essential oils from 12 species of Eucalyptus on Aedes aegypti larvae. Entomol. Exp. Appl. 2008, 129, 107-114. [CrossRef]

34. Bugarin, D.; Grbović, S.; Orčič, D.; Mitić-Ćulafić, D.; Knežević-Vukčević, J.; Mimica-Dukić, N. Essential oil of Eucalyptus gunnii Hook. as a novel source of antioxidant, antimutagenic and antibacterial agents. Molecules 2014, 19, 19007-19020. [CrossRef] [PubMed]

35. Caputo, L.; Smeriglio, A.; Trombetta, D.; Cornara, L.; Trevena, G.; Valussi, M.; Nazzaro, F. Chemical composition and biological activities of the essential oils of Leptospermum petersonii and Eucalyptus gunnii. Front. Microbiol. 2020, 11, 409. [CrossRef] [PubMed]

36. Elaissi, A.; Salah, K.H.; Mabrouk, S.; Larbi, K.M.; Chemli, R.; Harzallah-Skhiri, F. Antibacterial activity and chemical composition of 20 Eucalyptus species' essential oils. Food Chem. 2011, 129, 1427-1434. [CrossRef]

37. Brophy, J.J.; Lassak, E.V.; Toia, R.F. The steam volatile leaf oil of Eucalyptus pulverulenta. Planta Med. 1985, 51, 170-171. [CrossRef]

38. Zrira, S.; Bessiere, J.M.; Menut, C.; Elamrani, A.; Benjilali, B. Chemical composition of the essential oil of nine Eucalyptus species growing in Morocco. Flavour Frag. J. 2004, 19, 172-175. [CrossRef]

39. Ieri, F.; Cecchi, L.; Giannini, E.; Clemente, C.; Romani, A. GC-MS and HS-SPME-GC $\times$ GC-TOFMS determination of the volatile composition of essential oils and hydrosols (By-products) from four Eucalyptus species cultivated in Tuscany. Molecules 2019, 24, 226. [CrossRef]

40. Topiar, M.; Sajfrtova, M.; Pavela, R.; Machalova, Z. Comparison of fractionation techniques of $\mathrm{CO}_{2}$ extracts from Eucalyptus globulus-Composition and insecticidal activity. J. Supercri. Fluids 2015, 97, 202-210. [CrossRef]

41. Ramezani, S.; Saharkhiz, M.J.; Ramezani, F.; Fotokian, M.H. Use of essential oils as bioherbicides. J. Essent. Oil-Bear. Plants 2008, 11, 319-327. [CrossRef]

42. Kaur, S.; Pal Singh, H.; Batish, D.; Kumar Kholi, R. Role of monoterpenes in Eucalyptus communities. Curr. Bioact. Compd. 2012, 8, 101-107. [CrossRef]

43. Kohli, R.K.; Batish, D.R.; Singh, H.P. Eucalypt oils for the control of Parthenium (Parthenium hysterophorus L.). Crop Prot. 1998, 17, 122-199. [CrossRef]

44. Ibáñez, M.D.; Blázquez, M.A. Phytotoxicity of Essential Oils on Selected Weeds: Potential Hazard on Food Crops. Plants 2018, 7, 79. [CrossRef]

45. Romagni, J.G.; Allen, S.N.; Dayan, F.E. Allelopathic effects of volatile cineoles on two weedy plant species. J. Chem. Ecol. 2000, 26, 303-313. [CrossRef] 
46. Kaur, S.; Singh, H.P.; Batish, D.R.; Kohli, R.K. Chemical characterization and allelopathic potential of volatile oil of Eucalyptus tereticornis against Amaranthus viridis. J. Plant Interact. 2011, 6, 297-302. [CrossRef]

47. Murtaza, G.; Asghar, R. $\alpha$-Amylase activities during seed development and germination in pea (Pisum sativum L.) treated with salicylic acid. Pak. J. Bot. 2012, 44, 1823-1829.

48. Danabas, D.; Ates, M.; Tastan, B.E.; Cimen, I.C.C.; Unal, I.; Aksu, O.; Kutlu, B. Effects of Zn and ZnO nanoparticles on Artemia salina and Daphnia magna organisms: Toxicity, accumulation and elimination. Sci. Total Environ. 2020, 711, 134869. [CrossRef]

49. Council of Europe. European Pharmacopeia, 5th ed.; Council of Europe: Strasbourg, France, 2004; Volume I, pp. $217-218$.

50. Rogiers, S.; Hardie, W.J.; Smith, J.P. Stomatal density of grapevine leaves (Vitis vinifera L.) responds to soil temperature and atmospheric carbon dioxide. Aust. J. Grape Wine Res. 2011, 17, 147-152. [CrossRef]

51. O'Brien, T.P.; McCully, M.E. The Study of Plant Structure: Principles and Selected Methods; Termocarphy: Melbourne, Australia, 1981.

52. Chieco, C.; Rotondi, A.; Morrone, L.; Rapparini, F.; Baraldi, R. An ethanol-based fixation method for anatomical and micromorphological characterization of leaves of various tree species. Biotech. Histochem. 2013, 88, 109-119. [CrossRef] [PubMed]

53. Pathan, A.K.; Bond, J.; Gaskin, R.E. Sample preparation for scanning electron microscopy of plant surfaces-Horses for courses. Micron 2008, 39, 1049-1061. [CrossRef]

54. Schneider, C.A.; Rasband, W.S.; Eliceiri, K.W. NIH Image to ImageJ: 25 years of image analysis. Nat. Methods 2012, 9, 671-675. [CrossRef] [PubMed]

55. Jennings, W.; Shibamoto, T. Qualitative Analysis of Flavour and Fragrance Volatiles by Glass Capillary Gas Chromatography; Academic Press: New York, NY, USA, 1980.

56. Davies, N.W. Gas chromatographic retention indices of monoterpenes and sesquiterpenes on methyl silicone and Carbowax 20M phases. J. Chromatogr. 1990, 503, 1-24. [CrossRef]

57. Goodner, K.L. Practical retention index models of OV-101, DB-1, DB-5, and DB-Wax for flavor and fragrance compounds. LWT Food Sci. Technol. 2008, 41, 951-958. [CrossRef]

58. Adams, R.P. Identification of Essential Oil Components by Gas Chromatography/Mass Spectroscopy, 4th ed.; Allured Publishing Corporation: Carol Stream, IL, USA, 2007.

59. McLafferty, F.W. Wiley Registry of Mass Spectral Data, with NIST Spectral Data CD Rom, 7th ed.; John Wiley \& Sons: New York, NY, USA, 1998.

60. Bewley, J.D. Seed germination and dormancy. Plant Cell 1997, 9, 1055-1066. [CrossRef] [PubMed]

61. Xiao, Z.; Storms, R.; Tsang, A. A quantitative starch-iodine method for measuring alpha-amylase and glucoamylase activities. Anal. Biochem. 2006, 351, 146-148. [CrossRef] [PubMed]

62. Sudha, P.; Zinjarde, S.S.; Bhargava, S.Y.; Kumar, A.R. Potent $\alpha$-amylase inhibitory activity of Indian Ayurvedic medicinal plants. BMC Complement. Altern. Med. 2011, 11, 5. [CrossRef] 